\title{
miR-99a-3p Targeting ElF4EBP1 Affects B Lymphocytes Function Through Autophagy and Aggravates SLE Disease Progression
}

meng yang ( $\square$ yangmeng5@kmmu.edu.cn )

Kunming Medical University https://orcid.org/0000-0003-0629-8648

Deng Danqi

Dermatology Hospital of Southern Medical University: Guangdong Provincial Dermatology Hospital

\section{Research article}

Keywords: miR-99a-3p, EIF4EBP1, autophagy, B lymphocytes, SLE

Posted Date: July 14th, 2021

DOl: https://doi.org/10.21203/rs.3.rs-685987/v1

License: (c) (1) This work is licensed under a Creative Commons Attribution 4.0 International License.

Read Full License 


\section{Abstract}

Background:Overactivation of immune cells plays a key role in the pathogenesis of systemic lupus erythematosus (SLE). The regulation of immune cells by miRNA is a research hotspot.In this study, the second-generation high-throughput sequencing found that the expression of miR-99a-3p in SLE decreased, but the specific mechanism is still unclear.The purpose of this study is to explore the potential target genes, target cells of miR-99a-3p and their potential mechanisms affecting the progression of SLE.

Methods: Isolate PBMC from healthy individulas and SLE patients, transfect Ball-1, Jurkat, THP-1 and K562 cells with miR-99a-3p agomir and antagomir,detect miR-99a-3p, predict target genes and autophagy pathway mRNA and protein expression by RT-qPCR and Western blotting.CCK-8 method detects cell proliferation, PI method detects cell cycle, flow cytometry detects cell apoptosis, and luciferase reporter gene experiment determines miR-99a-3p target genes. With C57BL/6J mice as control,construct miR-99a$3 p$ overexpression and interference model based on MRL/Ipr mice,ELISA detects plasma ANA, dsDNA, IgE, IgM, IL-6, IL-10, BLyS.Pathological analysis of HE staining and C3 immunofluorescence(IF) deposition in mouse kidney tissue,Immunohistochemistry $(\mathrm{IHC})$ detects changes in target genes and pathway proteins in kidney tissue,isolate B cells to verify the differential expression of miR-99a-3p, target genes, pathway mRNA and protein.

Results: Compared with healthy individulas, miR-99a-3p in SLE was down-regulated, while EIF4EBP1, LC3II, LAMP-2A mRNA and protein expression were up-regulated.After Ball-1 was transfected with miR99a-3p agomir, cell proliferation decreased and apoptosis increased.After transfected with miR-99a-3p antagomir, the effect was opposite;Luciferase reporter gene detection proved that miR-99a-3p directly targets EIF4EBP1. Rescue experiments confirmed the interaction model between miR-99a-3p and EIF4EBP1. Clinical, in vitro, and in vivo experiments further confirmed that miR-99a-3p agomir can reduce the expression of EIF4EBP1, LC3-囚, and LAMP-2A, while miR-99a-3p antagomir had the opposite effect.In vivo experiment antagomir group mice serum ANA, dsDNA, IgE, IgM, IL-6, IL-10, BLyS were higher than those in the MRL/Ipr group, EIF4EBP1, LC3-X, LAMP-2A mRNA, protein and IHC levels also increased, and the urinary protein and C3 IF deposition of mice in the antagomir group were increased, and the related indexes of mice in the agomir group were lower than those in the MRL/Ipr group.

Conclusion:The expression of miR-99a-3p in SLE PBMC was down-regulated. Up-regulation of miR-99a$3 p$ by transfection can protect $B$ cells from autophagy mediated by EIF4EBP1.The down-regulation of miR-99a-3p induces autophagy by regulating the autophagy signaling pathway mediated by EIF4EBP1 in SLE B cells. These results indicate that miR-99a-3p and EIF4EBP1 may be potential targets of SLE.

\section{Introduction}

Systemic lupus erythematosus (SLE) is a type of autoimmune-mediated diffuse connective tissue disease that involves multiple systems throughout the body, characterized by pathological inflammation [1]. Studies had shown that genetic factors influence the clinical phenotype and 
progression of SLE, while environmental factors trigger the occurrence of SLE[2], and ultraviolet rays play an important role in stimulating SLE[3]. The age of onset of SLE in high altitude areas is lower, the course of disease is shorter. The proportion of patients with Anti-Sm positive, anemia, thrombocytopenia and elevated serum creatinine is significantly higher than that in low altitude areas [4].

The main ethnic group in the Diqing Tibetan Autonomous Prefecture in Yunnan Province is the Tibetans, they live in a high-altitude, low-oxygen, high-cold, dry and strong ultraviolet environment, relatively isolated, lack of gene exchange with the outside world. The epidemiological and transcriptomics characteristics of Tibetan SLE patients deserve in-depth and systematic research.

MicroRNAs (miRNAs) are a class of highly conserved endogenous non-coding single-stranded small RNAs with a length of about 21-25 nucleotides. More and more miRNAs had been found to play an important role in the pathogenesis of SLE[5]. Our research group found that UVB may be involved in the pathogenesis of SLE by decreasing miR-125b-5p of SLE and increasing the expression of target gene UVRAG and cell autophagy[6].

We collected venous blood from 10 Tibetan SLE patients and 10 healthy Tibetans. The miRNA was differentiated by high throughput sequencing of the second generation after RNA extraction (Fig. 1a). The sequencing results were verified by RT-qPCR, and it was found that miR-99a-3p in Tibetan SLE patients decreased significantly (Fig 1B).

As a member of the miR-99 family, miR-99a-3p is transcribed from the 21 region of the long arm of chromosome 21. miR-99 is expressed at low levels in a variety of human malignant tumors. It participates in the occurrence and development of a variety of urinary tumors[7], head and neck squamous cell carcinoma[8], liver cancer[9] and ovarian cancer[10], and has certain significance in the early diagnosis and staging of tumors[11].

Pradhan and Tomankova were the first to find that the expression of miR-99 in SLE decreased [12]. Jin et al found that the expression of miR-99a in South Korean SLE patients was down-regulated by miRNA PCR chip detection [13]. Frangou et al used cDNA microarrays to compare the gene expression in the effector cells and target tissues of SLE patients and control individuals, and found that miR-99a expression in SLE PBMC decreased, and it was related to the regulation of type I IFN pathway[14].There is no report on the expression of miR-99a-3p in SLE in the Chinese population.

Therefore, this study explores the role of miR-99a-3p in the pathogenesis of SLE. This study provides help to clarify the complex mechanisms involved in the pathogenesis of SLE and new targets for SLE.

\section{Materials And Methods}

\section{clinical experiments}

\section{SLE patients and healthy individulas included}


SLE patients: 10 cases of SLE patients of Han ethnicity who were treated in the outpatient clinic of the Second Affiliated Hospital of Kunming Medical University from January to December 2020, and 10 cases of SLE patients of Tibetan ethnicity in Diqing Tibetan Autonomous Prefecture. Both Tibetan and Han patients need to be diagnosed in accordance with the American Rheumatism Association's 1997 classification of SLE, and had not taken chloroquine or hydroxychloroquine in the past three months. The ethics was approved by the Ethics Committee of the Second Affiliated Hospital of Kunming Medical University (Trial-PJ-2020-135), and $20 \mathrm{ml}$ of peripheral venous blood was collected from patients and healthy individulas. There was no statistical difference in age or gender of each group $\triangle P>0.05$, General clinical data of study subjects see Supplementary Materials Table 1区.

\section{In vitro experiments}

\section{PBMC, primary $B$ cell separation and culture}

PBMC was selected by lymphocyte isolation fluid (LTS1077, Haoyang, Tianjin, China) for $20 \mathrm{ml}$ EDTA anticoagulant. RT-qPCR and Western blotting were used to detect PBMC and intervention after culture.

Take $1 \mathrm{ml}\left(1 \times 10^{8}\right)$ of separated PBMC in a $5 \mathrm{ml}$ test tube, add $200 \mathrm{ul}$ of MagniSort ${ }^{\mathrm{TM}}$ Enrichment Antibody Cocktail (Thermo, Germany) and mix well, incubate for $10 \mathrm{~min}$, centrifuge and discard the supernatant, add 200ul of MagniSort ${ }^{\mathrm{TM}}$ Negative Selection Beads (Thermo, Germany), mix and incubate, insert the test tube into the magnetic pole (Thermo, Germany), collect the supernatant, and centrifuge the precipitate to be B lymphocytes (identification of B cells and culture, see Supplementary Materials Fig 1.).

Take the frozen Ball-1, Jurkat, THP-1 and K562 cells (purchased from the Cell Bank of the Chinese Academy of Sciences) from the liquid nitrogen tank and dissolve them in a $37^{\circ} \mathrm{C}$ water bath, centrifuge to discard the supernatant, and add 1640 complete medium (containing $1 \%$ Double antibody $+10 \% \%$ FBS, Thermo, Germany) to resuspend and culture.

\section{RT-qPCR to detect the expression of miRNA/mRNA}

Add TRIzol ${ }^{\text {TM }}$ Reagent (15596-026, Lifetech, USA) lysate to extract total RNA, use SureScriptTM FirstStrand cDNA Synthesis Kit (Genecopoeia, Guangzhou, China) for mRNA, use All-in-One ${ }^{\text {TM }}$ miRNA FirstStrand cDNA Synthesis for miRNA Kit 2.0 (Genecopoeia, Guangzhou, China), take 1 ug of total RNA and synthesize the first-strand cDNA of mRNA/miRNA according to its instructions, mRNA at $25^{\circ} \mathrm{C}$ for $5 \mathrm{~min}$, $42^{\circ} \mathrm{C}$ for $45 \mathrm{~min}, 85^{\circ} \mathrm{C}$ for $5 \mathrm{~min}$, miRNA at $37^{\circ} \mathrm{C}$ for $60 \mathrm{~min}, 85^{\circ} \mathrm{Cunder} 5 \mathrm{~min}$ conditions, reverse transcription was performed on a common PCR machine (4359659, Applied Biosystems 2720 Thermal Cycler, USA). BlazeTaq ${ }^{\text {TM }}$ SYBR ${ }^{\circledR}$ Green qPCR Mix 2.0 (GeneCopoeia, Guangzhou, China) for mRNA, All-inOne miRNA RT-qPCR Detection System User Manual (GeneCopoeia, Guangzhou, China) for miRNA, using cDNA as a template and GAPDH/ U6 as the internal reference, using Sybrgreen method, detected on the CFX96 real-time quantitative fluorescent PCR instrument (Bio-Rad,USA).PCR amplification reaction conditions were: $95^{\circ} \mathrm{C} 10 \mathrm{~min}, 95^{\circ} \mathrm{C} 10 \mathrm{~s}, 60^{\circ} \mathrm{C} 20 \mathrm{sec}, 72^{\circ} \mathrm{C} 30 \mathrm{~s}$, for 40 cycles, collect and record the fluorescence, read the $C T$ value, and use the $2^{-\Delta \Delta C T}$ method to calculate the relative expression of the 
gene. The relevant primers were synthesized by Beijing Kinco Xinye Biotechnology Co., Ltd., and the sequence was shown in Table 2 of Supplementary Materials.

\section{miR-99a-3p agomir and antagomir transfect Ball-1, Jurkat, THP-1 and K562 cells}

miR-99a-3p agomir (Sense-CAAGCUCGCUUCUAUGGGUCUG, Antisense-CAGACCCAUAGAAG CGAGCUUG) and miR-99a-3p antagomir (Sense-CAGACCC AUAGAAGCGAGCUUG) were synthesized by Ribobio Co. (Guangzhou, China). When the cell confluence reached 80\%, 2ul lipo2000 transfection reagent (Thermo, Germany) was added for transient transfection, the medium was changed after $6 \mathrm{~h}$ of transfection, and RT-qPCR was performed on the cells after $48 \mathrm{~h}$ of culture.

\section{CCK-8 detection method to detect cell proliferation}

Inoculate newly cultured Ball-1, Jurkat, THP-1, and K562 cell suspensions (100ul/well) in 96-well plates, and transfect miR-99a-3p agomir and antagomir into four cell lines respectively. Add 10ul CCK8 (Life iLab Biotech Co., Shanghai, China) to each well at $24 \mathrm{~h}, 48 \mathrm{~h}, 72 \mathrm{~h}, 96 \mathrm{~h}$, incubate for 2 hours, and measure the absorbance at $450 \mathrm{~nm}$ with a microplate reader (BioTek, USA).

\section{PI method to detect cell cycle}

Take 250 ul ( $1 \times 10^{6}$ cells) cell suspension, add 750 ul of pre-cooled absolute ethanol, seal and place at $-20^{\circ} \mathrm{C}$ for fixation overnight; add $500 \mathrm{ul} \mathrm{PI} \mathrm{staining} \mathrm{solution} \mathrm{(Becton,} \mathrm{Dickinson} \mathrm{and} \mathrm{Company,} \mathrm{USA)} \mathrm{to} \mathrm{a}$ final concentration of $65 \mathrm{ug} / \mathrm{ml}$, Incubate at $37^{\circ} \mathrm{Cfor} 30$ minutes, and immediately perform flow cytometric detection (Thermo, Germany). ModFit LT ${ }^{\mathrm{TM}} 4.1$ software was used to analyze cell cycle.

\section{FITC Annexin V method to detect cell apoptosis}

According to the apoptosis test kit Annexin V FITC Apop Dtec Kit I (556547, BD, USA) instructions, use 300ul $1 \times$ Binding Buffer cells at a concentration of $1 \times 10^{6} / \mathrm{ml}$, add $5 \mathrm{ul}$ Annexin V-FITC and Propidium lodide Staining Solution staining, add Binding Buffer, light incubate, and analyze on Attune NxT Streaming Cytometer (Invitrogen, USA).

\section{Dual-Luciferase Reporter Assay}

The miR-99a-3p target genes were predicted, and EIF4EBP1, NCAPG, IKBKB, PRKCB were selected for luciferase detection. The related target gene 3'UTR Wild/Mutant type luciferase vector pmirGLO Vector was constructed by Wuhan GeneCreate Company. Take miRNA and plasmid 2ug incubated for $5 \mathrm{~min}$, and add 2ul lipo2000 transfection reagent to culture medium for incubation. When the cell fusion degree reaches $80 \%$, add $500 \mathrm{ul}$ of the above transfection complex, add the extracted protein to $100 \mathrm{ul}$ of firefly luciferase substrate, measure its firefly luciferin value, add Renilla luciferase substrate, and measure the sea level. Kidney fluorescence value. The renilla fluorescence value of each well divided by the firefly fluorescein value was the fluorescence value of the reporter plasmid in each well. 


\section{Western-blotting}

Add 250ul RIPA lysis buffer (containing protease inhibitor, Thermo, Germany) to each group of cells to be tested, and BCA protein quantification kit (P0010, Beyotime, China) to determine the protein concentration. Take 30ug of total protein for SDS-PAGE electrophoresis, and block after transfer to membrane. Add the primary antibodies EIF4EBP1 (1:1000, GTX133182, GeneTex, USA), LAMP-2A (1:1000, ab24170, Abcam, USA), LC3B (1:1000, ABS1513, Millipore, USA), B-Actin (LMAI Bio,Shanghai, China), shake overnight at $4^{\circ} \mathrm{C}$, rewarm for one hour the next day, add secondary antibody (Peroxidase labeled goat anti-rabbit IgG, 1:5000, Sigma, USA), incubate for 30min, exposure instrument (Monad, Suzhou, China), add ECL color developing solution (Thermo, Germany) to take pictures, use Image-Pro Plus 6.0 software to analyze the optical density of the stripe, the optical density ratio of the target protein and the light density ratio of the endoprotein $\beta$-actin represents the relative content of the target protein to compare the difference in protein expression.

\section{SiRNA and antagomir jointly intervene in Ball-1 cells for Rescue experiment}

siRNA EIF4EBP1 was synthesized by Guangzhou Ruibo Biological Company, and transfected Ball-1 cells with siEIF4EBP1-1, siEIF4EBP1-2, and siEIF4EBP1-3. After 48 hours, RT-qPCR and Western-blotting were detected. It was found the expression of EIF4EBP1 in the siEIF4EBP1-1 group was lower than siNC, the difference was statistically significant (sequence and screening see supplementary material Table3,Fig 2). Collect Ball-1 cell count and plate, add siRNA EIF4EBP1 and NC respectively, and then add lipo2000 transfection reagent. After $24 \mathrm{~h}$, half of the sample was separated from the siRNA tube and added miR$99 a-3 p$ antagomir for further culture. After incubation at $37^{\circ} \mathrm{Cfor} 48$ hours, proliferation, cycle, apoptosis and Western blotting were detected.

\section{In vivo experiment}

\section{miR-99a-3p over-expression and interference with MRL/lpr mouse model building}

Take 18 MRL/Ipr mice (20-30g, female, 6-8 weeks old, purchased from SPF Biotechnology Co., Ltd.), and group them into a single high-dose tail vein injection of miR-99a-3p agomir, antagomir and NC, injection volume $200 \mu \mathrm{l}$, miR-99a-3p agomir/antagomir injection doses were $20 \mathrm{nmol} / 200 \mathrm{nmol}$ respectively[15, 16].6 C57BL/6J (C57) mice (20-25g, female, 6-8 weeks old) purchased from Hunan Slack Jingda Experimental Animal Co., Ltd.

Eyeball venous blood was collected, and B lymphocytes were separated by immunomagnetic bead method, followed by Western-blotting, RT-qPCR, and plasma retention for ELISA . Mice were sacrificed by neck breaking method, and the kidneys were removed in layers and placed in $4 \%$ paraformaldehyde (P0099, Beyotime, China) for fixation. The experimental protocol was approved by the Animal Research Committee of Kunming Medical University (kmmu2021724).

\section{Coomassie brilliant blue method for quantitation of total protein in the urine}


Take appropriate amount of standard dilution, and take appropriate amount of urine to be measured, PBS equal multiple dilution, add $5 \mathrm{ml}$ dilution of the Coomassie brilliant blue solution (Xinfan Biological Biological Technology Co., Shanghai, China), the color changes from red to blue, the absorbance was determined at $595 \mathrm{~nm}$.

\section{ELISA detects ANA, dsDNA, IgE, IgM, IL-6, IL-10, BLyS}

Take out the ELISA kit (JL12477-96T, Jiang Lai Bio, China) slats, add 100 $\mu \mathrm{L}$ of HRP-labeled antibody, incubate at $37^{\circ} \mathrm{C}$ for $60 \mathrm{~min}$, wash the plate; add substrate $A$ and B $50 \mu \mathrm{l}$ to each well , then incubate at $37^{\circ} \mathrm{Cin}$ dark incubation and add the stop solution. Measure the OD value of each well at $450 \mathrm{~nm}$ wavelength, and calculate the sample concentration by the absorbance value of the sample and the standard curve.

\section{HE staining}

Take the longitudinally sectioned kidney tissues of each group and fix them in paraformaldehyde for 24 hours, and then put them in a low-to-high concentration ethanol solution for dehydration, transparency, wax immersion, embedding, and cut into slices with a thickness of about 4 um, and bake at $64^{\circ} \mathrm{Cfor} 30$ minutes. After dewaxing with xylene, put the slides in the ethanol solution of high concentration to low concentration to gradually hydrate, counterstain with hematoxylin (C0105-1, Beyotime, China) for 4 minutes, rinse with distilled water, and put it into alcohol-hydrochloric acid solution for differentiation, return to blue in tap water for 20 minutes, stain with eosin (C0109, Beyotime, China) for 10 seconds, and wash with distilled water. Finally, use ethanol dehydration, transparent xylene and neutral gum to mount the slides, take pictures with a microscope (Lab.A1, ZEISS, Germany) and analyze the staining results.

\section{Immunohistochemical staining}

Prepare slices, bake slices, and dewax.After hydration with gradient alcohol, add $0.01 \mathrm{M}$ citric acid buffer and boil for 15 minutes to restore the antigen, block with 5\% BSA (LMAI Bio, Shanghai, China) at room temperature for 30 minutes, add primary antibody EIF4EBP1 (1:100, GTX133182, GeneTex, USA), LAMP2A (1:100, ab24170, Abcam, USA), LC3B (1:100, ABS1513, Millipore, USA) overnight at $4^{\circ} \mathrm{C}$, dropwise reaction Incubate with the enhancement solution, add the secondary antibody (Sheep anti-mouse, A21235, Invitrogen) and incubate at $37^{\circ} \mathrm{C}$ for 30 minutes, add the DAB (ZLI-9019, Zhongshan Golden Bridge, China) dye solution dropwise to the tissue block for 5 minutes, and place the slide on the Stained in hematoxylin for 4 minutes, and the tap water turned blue for 20 minutes; dehydrated, transparent, and photographed after mounting, scanning the positive area and calculating the positive rate.First score according to the staining intensity: 0 is divided into colorless, 1 is divided into light yellow, 2 is divided into brown and 3 is divided into brown, and the staining intensity needs to be compared with the background coloring;Then score according to the percentage of positive cells: 0 is negative, 1 is positive cells $\leq 25 \%, 2$ is $25 \%-50 \%$, and 3 is $>50 \%$. 
Prepare slices, bake slices, dewax, and put them in the repair solution after hydration with gradient alcohol; draw circles with oily brushes, add diluted serum, Anti-C3 antibody (1:100, ab11887, Abcam, USA) incubate overnight and then add secondary antibody Goat Anti-Rabbit IgG H\&L (AmyJet Scientific, Wuhan, China), drop DIPA (Weifang Bincheng Chemical Industry) dyeing solution.Put it under a fluorescence microscope (Mshot, Guangzhou, China), and take photos of a total of 5 fields of view in the center and surrounding areas of each film, and calculate the positive rate.

Collect the cells and fix them with 4\% paraformaldehyde. After blocking, add the 1:200 diluted primary antibody LC3B (1:100, ABS1513, Millipore, USA) and incubate overnight. Then add the secondary antibody (Sheep anti-mouse, A21235, Invitrogen), and then add DAPI (ID0080, Solarbio, China) to incubate, mount, take pictures, and calculate the positive rate.

\section{Statistical methods}

The data were expressed as Mean $\pm S D$, analyzed by ANOVA and LSD-t test, and statistically analyzed by SPSS 23.0 software. Two tailed $P<0.05$ was statistically significant. The correlation analysis used Spearman's rank correlation analysis, the count data used the $\chi^{2}$ test. GraphPad Prism 6.0 is used for statistical analysis of histograms.

\section{Results}

\section{Differential verification of miR-99a-3p in SLE}

The venous blood of Tibetan SLE patients and healthy individulas was collected, and RNA was extracted and then subjected to second-generation high-throughput sequencing. RT-qPCR verified the sequencing results which shows miR-99a-3p in Tibetan SLE patients had decreased significantly (Fig 1A, B).

RT-qPCR detected miR-99a-3p in PBMCs of 10 Tibetan\&Han SLE patients and 10 Tibetan\&Han healthy individulas. The miR-99a-3p in both Tibetan and Han SLE patients was decreased compared with the healthy controls, with statistical significance(Fig $1 \mathrm{C}$ and D).

\section{Functional difference of miR-99a-3p on Ball-1, Jurkat, THP-1, K562 cells}

The synthesized miR-99a-3p agomir and antagomir were transfected into Ball-1, Jurkat, THP-1, K562 (see Supplementary Material Fig 1 for cell morphology), and the expression of miR-99a-3p was detected by RTqPCR 48h after transfection. Compared with the NC group, miR-99a-3p increased after miR-99a-3p agomir transfected with the four cell lines, and the difference was statistically significant. miR-99a-3p decreased after miR-99a-3p antagomir transfected the four cell lines, the difference was statistically significant (Fig 2A).

After Ball-1 and THP-1 were transfected with miR-99a-3p agomir, cell proliferation decreased on 1, 2, 3, and 4 days compared with NC group;Ball-1 and THP-1 were transfected with miR-99a-3p antagomir, cell proliferation increased on 1, 2, 3, and 4 days compared with NC group, and the difference was statistically 
significant; Jurkat and K562 were transfected with agomir and antagomir, cell proliferation had no significant changes (Fig 2B).

After Ball-1, Jurkat, THP-1 were transfected with miR-99a-3p agomir, the apoptosis rate was significantly higher than that of NC group; Ball-1, Jurkat were transfected with miR-99a-3p antagomir, the apoptosis rate was significantly lower than that of NC group, and the difference was statistically significant.After K562 transfection,the apoptosis rate of agomir and antagomir groups did not change significantly compared with NC group (Fig 2C).

After Ball-1 and THP-1 transfected miR-99a-3p agomir, the number of cells in G0/G1 phase was higher than that of NC group; Ball-1 and THP-1 transfected miR-99a-3p antagomir, the number of cells in G0/G1 phase was lower than that of NC group, and the difference was statistically significant.

After Ball-1 transfected miR-99a-3p agomir, the number of cells in G2/M and S phase decreased compared with NC group;after transfected miR-99a-3p antagomir, the number of cells in G2/M and $S$ phase was higher. After Jurkat transfected miR-99a-3p antagomir, the number of cells in G2/M phase was lower than that in NC group $(P<0.05)$. After $\mathrm{K} 562$ transfected, the agomir and antagomir groups had no significant changes in each cycle compared with the NC group (Fig 2D). The cell proliferation, apoptosis and cycle changes of the Ball- 1 line were relatively stable after transfected miR-99a-3p agomir, antagomir and NC.

\section{Target gene confirmation}

The target genes of miR-99a-3p were predicted by database囚TarBase,http://mirtarbase.mbc.nctu. edu.tw/php/index.php,miRDB,http://www.mirdb.org,Targetscan, http://www.targetscan.org/vert_71,

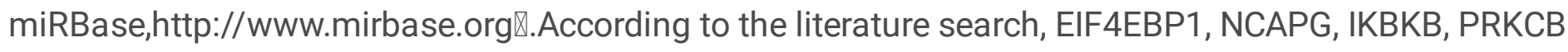
were selected as the research objects.

RT-qPCR detected the expression of EIF4EBP1, NCAPG, IKBKB, and PRKCB in the PBMC of Han SLE patients and Han healthy individulas. The expression of related genes was up-regulated in the PBMC of SLE patients, and the difference was statistically significant (Fig 3A).

Ball-1 cells were transfected with miR-99a-3p agomir, antagomir, and NC, and the target gene expression was detected by RT-qPCR 48h after transfection. Compared with NC group, the target genes all decreased after miR-99a-3p agomir transfection. The target genes all increased after miR-99a-3p antagomir transfection, and the difference was statistically significant (Fig 3B).

Hsa-miR-99a-3p+EIF4EBP1-WT was transfected into 293T cells.Compared with NC+EIF4EBP1-WT group, the fluorescence intensity was significantly reduced $(P=0.0055)$; while Hsa-miR-99a-3p+EIF4EBP1 -MT was transfected compared with the NC+EIF4EBP1-MT group, the fluorescence intensity did not change significantly after staining 293T cells $(P=0.5411)$, and the predicted position was the target relationship between Hsa-miR-99a-3p and EIF4EBP1 (Fig 3C). 
Hsa-miR-99a-3p+NCAPG-WT was transfected into 293T cells. Compared with NC+NCAPG-WT group, the fluorescence intensity was significantly reduced ( $P=0.0002)$; while Hsa-miR-99a-3p+NCAPG -MT was transfected compared with the NC+NCAPG-MT group, the fluorescence intensity of 293T cells did not change significantly $(P=0.0712)$, and the predicted position was the target relationship between Hsa-miR99a-3p and NCAPG. Hsa-miR-99a-3p had no targeting relationship with IKBKB and PRKCB (Fig 3D).

Western blotting further verified the expression of target genes in SLE and healthy individulas. The expression of EIF4EBP1 and NCAPG in SLE increased, and the difference was statistically significant (Fig 3E).

\section{miR-99a-3p participates in autophagy signaling pathway through target genes}

RT-qPCR detected the expression of LC3-Wand LAMP-2A in the PBMC of SLE and healthy individulas. SLE was higher than that of healthy control group, and the difference was statistically significant (Fig 4A). Western blotting analyzed SLE and healthy individual PBMC autophagy pathway marker protein LC3-

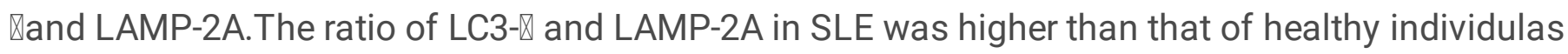
$(P=0.031$, Fig 4B).

miR-99a-3p agomir, antagomir, NC transfected Ball-1, the expression of LC3-II and LAMP-2A was detected by RT-qPCR 48h after transfection. After miR-99a-3p agomir was transfected with Ball-1, both LC3- $\triangle$ and LAMP-2A decreased, and the difference was statistically significant. The result was opposite after miR99a-3p antagomir transfection (Fig 4C).

Compared with NC group, miR-99a-3p increased after miR-99a-3p agomir transfected with B cells

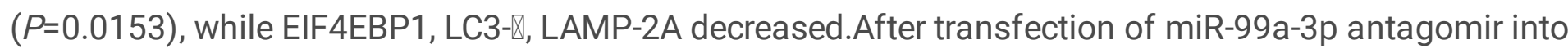

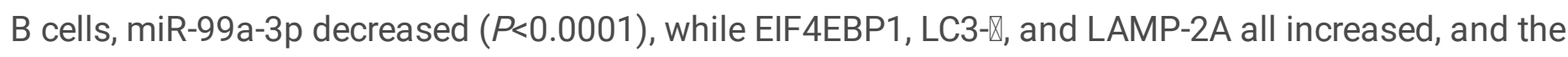
difference was statistically significant (Fig 4D).

48h after miR-99a-3p agomir, antagomir, and NC transfected Ball-1 and B cells, compared with the NC group, the EIF4EBP1, LC3- $\$ and LAMP-2A proteins in the miR-99a-3p agomir group were decreased, and the difference was statistically significant. The transfection result of miR-99a-3p antagomir group was opposite (Fig 4E).

Fluorescence microscope was used to observe the IF of LC3- $\triangle$ after transfection. The IF of LC3- $₫$ in the miR-99a-3p agomir group in Ball-1 and B cells was lower than that in NC group. The LC3-II IF of the miR99a-3p antagomir group was higher than that of NC group (Fig 4F).

\section{Ball-1 function changes after Rescue experiment}

Western blotting was used to detect the expression of EIF4EBP1, LC3-囚, LAMP-2A after siNC, siEIF4EBP1,

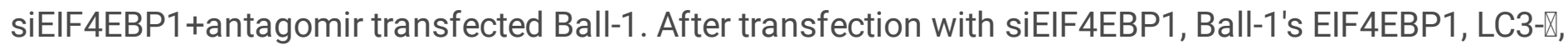
LAMP-2A protein expressions were all lower than that of the siNC group. After transfection with 
siEIF4EBP1+antagomir, the protein expressions of EIF4EBP1, LC3-X, and LAMP-2A in Ball-1 were higher than those in the siEIF4EBP1 group, and the difference was statistically significant (Fig 5A).

Flow cytometry was used to detect the difference in cell apoptosis after transfection of Ball-1. After Ball-1 was transfected with siEIF4EBP1, the apoptotic rate was significantly higher than that of the siNC group; after transfection with siEIF4EBP1+antagomir,the apoptosis rate was significantly lower than that of the siEIF4EBP1 group, and the difference was statistically significant (Fig 5B).

After Ball-1 transfection, the cell proliferation of the siEIF4EBP1 group at 1, 2, 3, and 4 days was lower than that of the siNC group; the cell proliferation of the siEIF4EBP1+antagomir group at 2, 3, and 4 days was higher than that of the siEIF4EBP1 group.The difference was statistically significant (Fig 5C).

After transfection with siEIF4EBP1, the number of cells in the G0/G1, G2/M phase was higher than that in siNC group, and the number of cells in the $S$ phase was less than that in siNC group. After transfection with siEIF4EBP1+antagomir, the number of cells in the G0/G1 phase decreased compared with the siEIF4EBP1 group, and the number of cells in the G2/M and $S$ phase increased and the cell proliferation recovered (Fig 5D). Fluorescence microscope was used to observe the LC3-II IF of siNC, siEIF4EBP1, siEIF4EBP1+antagomir transfected with Ball-1. It was found that the LC3-II IF of siEIF4EBP1 group was lower than that of siNC group, and the LC3-II IF of siEIF4EBP1+antagomir group was higher than that of siEIF4EBP1 group. The difference was statistically significant (Fig 5E).

\section{Disease progression of MRL/Ipr lupus mice after experimental intervention of miR-99a-3p in vivo}

The body weight of the four groups of mice increased with the increase in the number of feeding weeks. One mouse in the agomir group died at $12 \mathrm{w}$, and the body weight of the MRL/Lpr group was higher than that of $\mathrm{C} 57$ at $10 \mathrm{w}, 12 \mathrm{w}$, and $13 \mathrm{w}$. However, miR-99a-3p intervention did not significantly change the body weight (Fig 6A).At 13w, the hair around the nose and eyes of the MRL/Ipr group decreased,and there was fewer and slower behavioral activities,the antagomir group mice had alopecia area around the nose, eyes and forehead, and the behavioral activity was obviously slow.

There was no difference in urine protein between the four groups at 8 weeks $(P>0.05)$. However, at $10 \mathrm{w}$, $12 w$, and $13 w$, the urine protein in the MRL/Lpr, agomir, and antagomir group increased significantly. The urine protein of the MRL/Lpr group was higher than that of the C57 group. The urine protein in the antagomir group was higher than that of the $\mathrm{C} 57$ group; the urine protein in the agomir group was lower than that of the C57 group, and the difference was statistically significant (Fig 6B).

CaseViewer 3.3 counted the glomerular area,the glomerular area of the MRL/Ipr group was higher than that of the $\mathrm{C} 57$ group $(P=0.0184)$, suggesting that the mice in the MRL/Ipr group had some glomerular edema. The glomerular area of the agomir group was lower than that of the MRL/Ipr group, but the difference was not statistically significant $(P=0.2098)$. The glomerular area of the antagomir group was higher than that of the MRL/Ipr group, but the difference was not statistically significant $(P=0.5888)$ (Fig $6 C, D)$. 
The C3 IF deposition of MRL/Ipr mice and C57 mice after intervention was calculated, and it was found that the C3 IF deposition of the MRL/Ipr group was higher than that of the C57 group $(P<0.0001)$. C3 deposition in the agomir group was lower than that in the MRL/Ipr group $(P=0.0002)$. C3 deposition in the antagomir group was higher than that in the MRL/Ipr group ( $P=0.0008$, Fig $6 \mathrm{E}, \mathrm{F})$.

ELISA detection found that the levels of ANA, dsDNA, IgE, IgM, IL-6, IL-10, and BLyS in the MRL/Ipr group were higher than those in the C57 group.The levels of ANA, dsDNA, IgE, IgM, IL-6, IL-10 and BLyS in the agomir group were lower than those in the MRL/Ipr group. The levels of ANA, dsDNA, IgE, IgM, IL-10, and BLyS in the antagomir group were higher than those in the MRL/Ipr group, and the difference was statistically significant (Fig 6G).

\section{Changes of target genes and pathway proteins in MRL/lpr lupus mice after experimental intervention of miR-99a-3p in vivo}

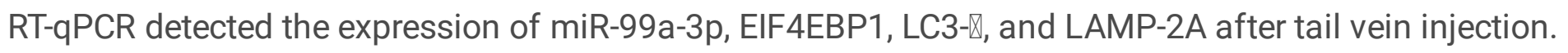
The expression of miR-99a-3p in the MRL/Ipr group was lower than that in the $\mathrm{C57}$ group ( $P=0.0293)$. The expression of miR-99a-3p in agomir group was higher than that in MRL/Ipr group ( $P=0.0013)$. The expression of miR-99a-3p in the antagomir group was lower than that in the MRL/lpr group $(P=0.0272)$. The expression of EIF4EBP1, LC3- $\triangle$ and LAMP-2A in the MRL/Ipr group was higher than that in the C57 group. The mRNA expression of EIF4EBP1, LC3- $\triangle$ and LAMP-2A in agomir group was lower than that in $\mathrm{MRL} / \mathrm{lpr}$ group. The expression levels of EIF4EBP1, LC3-囚, and LAMP-2A mRNA in the antagomir group were higher than those in the MRL/Ipr group, and the difference was statistically significant (Fig 7A).

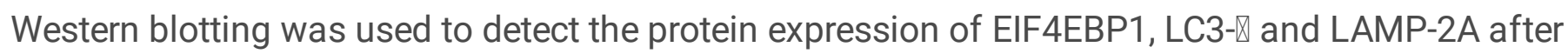
intervention in MRL/Ipr and C57 mice. The expression levels of EIF4EBP1, LC3-囚, and LAMP-2A in the $\mathrm{MRL} / \mathrm{Ipr}$ group were higher than those in the $\mathrm{C} 57$ group $(P=0.0293)$. The expression of EIF4EBP1, LC3- $\mathbb{Z}$ and LAMP-2A in agomir group was lower than that in MRL/lpr group $(P=0.0013)$. The expression of EIF4EBP1, LC3- $₫$ and LAMP-2A in the antagomir group was higher than that in the MRL/Ipr group $(P=0.0272)$ (Fig 7B).

IHC staining showed that the expression of EIF4EBP1, LC3-囚, LAMP-2A in the kidney of C57 mice was weak, while the MRL/Ipr group increased, the antagomir group increased much more significantly, and in the agomir group significantly weakened (Fig 6C). EIF4EBP1, LC3-囚, LAMP-2A in the MRL/Ipr group were lower than the $\mathrm{C} 57$ and agomir groups at 0 points, and higher than the antagomir group, the difference was statistically significant. As the score increased, the intensity of IHC staining and the percentage of positive cells in the $\mathrm{C} 57$ and agomir groups gradually decreased, while the trend in the antagomir group was opposite (Fig 7C).

\section{Discussion}

SLE is an immune system disease caused by over-activation of immune cells and massive secretion of autoantibodies. Extremely active B cells are involved in almost all the pathogenesis of SLE. Although the 
treatment of SLE has made great progress, there are still patients with ineffective treatment or relapse.

miRNA is a type of endogenous non-coding RNA widely distributed in the human body, which plays an important role in regulating cell proliferation, apoptosis and disease progression. MiRNAs are involved in immune disorders and organ damage in SLE. MiRNA-based biomarkers and treatment methods may become viable options for the treatment of SLE[5].

Zhang et al provide a novel insight into the role of the circRNA-miRNA-mRNA regulation network in the SLE and 29 DECs (2 up and 27 down) of SLE were found[17]. Latini et al found that miR-155, miR-499a and miR-142 are involved in the pathogenesis and clinical phenotype of SLE[18]. Tao et al found that miR-152-3p promotes TLR-mediated CD4+T cell inflammatory response by regulating the DNMT1/MyD88 signaling pathway, which may become a new target for SLE treatment[19].

This study found that miR-99a-3p decreased significantly in Tibetan and Han SLE patients, which was consistent with Pradhan's report on the Indian population[12], Jin's report on the Korean population[13], and Frangou's report on the European population[14].There was no report on the expression of miR-99a$3 p$ in SLE in Chinese population. This study aimed to explore the functional mechanism of miR-99a-3p. MiR-99a-3p decreased in both Tibetan and Han SLE patients. The Han SLE patients who are more convenient to obtain were selected as further research objects.

In order to clarify the function of miR-99a-3p in different immune cells, this study selected Ball-1, Jurkat, THP-1, K562 cell lines for functional verification. After Ball-1 transfection of miR-99a-3p agomir, antagomir, NC, cell proliferation, apoptosis and cycle changes were relatively stable. In this experiment, Ball-1 was selected as the research object. In the pathogenesis of SLE, B cells not only produce autoantibodies, but also regulate the activation of $T$ cells through various cytokines and antigen presentation processes, which also aggravates the progress of SLE[20]. Therefore, it is particularly important to study how B cells play a role in SLE and how to produce pathogenic auto antibodies through signal transmission between cells.

In order to further study the specific molecular mechanism of miR-99a-3p functioning, it was predicted that EIF4EBP1 was the target gene of miR-99a-3p through bioinformatics methods. In this study, 293T cells were transfected to construct the luciferase reporter gene vector of the 3'UTR wild-type/mutant region of EIF4EBP1, which confirmed that miR-99a-3p can combine with the 3'UTR complementary sequence of EIF4EBP1 and down-regulate EIF4EBP1.

EIF4EBP1 is a translation initiation inhibitor, which regulates its activity by preventing the assembly of elF4E into the elF4F complex: hypophosphorylated form competes with EIF4G1/EIF4G3 and strongly binds to EIF4E, leading to repress translation. In contrast, hyperphosphorylated form dissociates from EIF4E, leading to initiation of translation[21]. The pathogenesis of EIF4EBP1 and SLE had not been reported. 
Autophagy is a process of engulfing one's own cytoplasmic proteins or organelles, coating them into vesicles, and then fusing with lysosomes to degrade the contents of the package. A large number of studies had confirmed that miRNA can regulate autophagy[22, 23]. Abnormal autophagy function leads

to the accumulation of apoptosis and induces the production of autoantibodies, thereby inducing and aggravating the condition of SLE.EIF4EBP1 can regulate MTORC1 to induce autophagy[24], microtubulassociated protein 1 light chain 3 (LC3) is a key protein involved in autophagy[25]. The lysossomal associated protein $2 \mathrm{a}$ (LAMP-2A) is a key regulatory protein in the chaperonmediated autophagy (CMA) pathway. Inhibiting the LAMP-2A protein can specifically block the CMA pathway[26].

This study further confirmed that transfection of miR-99a-3p agomir reduced the expression of

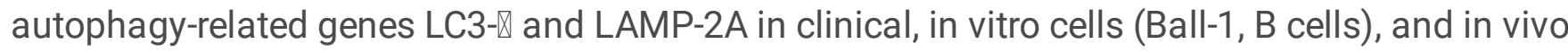
experiments (MRL/Ipr mouse B cells).Transfection of miR-99a-3p antagomir had the opposite effect, suggesting that miR-99a-3p had a negative regulatory effect on autophagy, and the change trend of EIF4EBP1 is consistent with the change trend of autophagy level, suggesting that EIF4EBP1 had a positive regulatory effect on autophagy.

In this study, rescue experiment was carried out to confirm the interaction model of miR-99a-3p and EIF4EBP1, miR-99a-3p affects cell proliferation and apoptosis through target genes.

B lymphocyte stimulator (BLyS) is a new member of the tumor necrosis factor family, involved in the regulation of $B$ cell proliferation, differentiation and antibody production. Transgenic mice overexpressing BLyS increased the number of B cells, increased serum ANA and dsDNA, and deposited immunoglobulin in the kidney[27]. Benlysta was the first inhibitor to act on BLyS, which binds to soluble BLyS with high affinity and inhibits its activity to achieve disease control[28].

In this study, an in vivo experimental model of MRL/Lpr was constructed by injecting miR-99a-3p agomir, antagomir, and NC into the tail vein. The ANA, dsDNA, IgE, IgM, IL-6, IL-10, and BLyS of mice in the antagomir group were significantly increased, SLE disease activity was stronger, and B cell proliferation and differentiation actively produced more antibodies. In the antagomir group, the urinary protein and C3 IF deposition in the kidneys were increased, and the kidney damage was more serious, consistent with reports in the literature[29]. They all reflected that miR-99a-3p inhibition increased the progression of SLE disease. However, the IgM level of mice in the antagomir group was higher than that of the MRL/lpr group, and the IgM level was not negatively correlated with the severity of SLE disease[30]. This may be related to the 13-week-old when the mice were sacrificed in this study, and they were still in the early stage of negative feedback.

\section{Conclusions}

This study first discovered that miR-99a-3p targeting EIF4EBP1 participates in the autophagy signaling pathway and affects the function of B cells, thereby aggravating the progression of SLE. This study had deepened the understanding of the molecular mechanisms of miRNA regulatory networks related to the 
pathogenesis of SLE.The abnormally expressed miR-99a-3p and its target gene EIF4EBP1 found in the study are expected to become potential targets of SLE.

\section{Limitations}

There are some limitations in this study. First, most of the experiments in this study were done on Ball-1 and B cells, but there are still some differences between Ball-1, B cells of healthy individulas and B cells of SLE patients. The results of the B cells in this study were not ideal, and the research was not in-depth. Second, the rescue experiment did not use gene knockout mice and lacked relevant functional verification experiments. Third, due to various restrictions, Tibetan patients were not directly involved in the research. The number of clinical cases was small, and the correlation analysis between clinical indicators and laboratory indicators was lacking.

\section{Declarations}

\section{Competing interests}

The authors have no proprietary interest in any aspect of the study.

\section{Funding}

This work was supported by the National Natural Science Foundation of China (grant number 81860552,31860256).

\section{Authors' information}

1.Department of Dermatology, the Second Affiliated Hospital of Kunming Medical University, 374 Miandian Rd., Kunming 650101, Yunnan, China. 2.Department of Dermatology, the Third Affiliated Hospital of Guangxi Medical University, 13 Dancun Rd., Nanning 530031, Guangxi, China

\section{Availability of data and materials}

All datasets generated for this study are included in the article/Supplementary Material.

\section{Authors' contributions}

Deng Danqi conceived and designed the experiments,Yang Meng performed the experiments and analyzed the results of the experiments.

\section{Acknowledgements}

We thank Yanlin Zi for help with the English Language polishing.

\section{Ethics approval and consent to participate}


Not applicable.

\section{Patient consent for publication}

Not applicable.

\section{References}

1. Lf A, Djw A, Cja B: The lung in systemic lupus erythematosus - ScienceDirect. Systemic Lupus Erythematosus (Second Edition) 2021:427-438.

2. Gulati G, Brunner HI: Environmental triggers in systemic lupus erythematosus. Semin Arthritis Rheum 2018, 47(5):710-717.

3. Hile GA, Kahlenberg JM: Immunopathogenesis of skin injury in systemic lupus erythematosus. Curr Opin Rheumatol 2021, 33(2):173-180.

4. Qian G, Ran X, Zhou CX, Deng DQ, Zhang PL, Guo Y, Luo JH, Zhou XH, Xie H, Cai M: Systemic lupus erythematosus patients in the low-latitude plateau of China: altitudinal influences. Lupus 2014, 23(14):1537-1545.

5. Hong S-M, Liu C, Yin Z, Wu L, Qu B, Shen N: MicroRNAs in Systemic Lupus Erythematosus: a Perspective on the Path from Biological Discoveries to Clinical Practice. Curr Rheumatol Rep 2020, 22(6):17.

6. Cao W, Qian G, Luo W, Liu X, Pu Y, Hu G, Han L, Yuan L, Xiao A, Deng D: miR-125b is downregulated in systemic lupus erythematosus patients and inhibits autophagy by targeting UVRAG. Biomedicine \& Pharmacotherapy 2018, 99:791-797.

7. Tsai T-F, Chen P-C, Lin Y-C, Chou K-Y, Chen H-E, Ho C-Y, Lin J-F, Hwang TIS: Benzyl isothiocyanate promotes miR-99a expression through ERK/AP-1-dependent pathway in bladder cancer cells. Environ Toxicol 2020, 35(1):47-54.

8. Okada R, Koshizuka K, Yamada Y, Moriya S, Kikkawa N, Kinoshita T, Hanazawa T, Seki N: Regulation of Oncogenic Targets by (Passenger Strand of Duplex) in Head and Neck Squamous Cell Carcinoma. Cells 2019, 8(12):1535.

9. Tao C, Sun H, Sang W, Li S: miRNA-99a inhibits cell invasion and migration in liver cancer by directly targeting. Oncol Lett 2019, 17(6):5108-5114.

10. Zhang L, Liu XL, Yuan Z, Cui J, Zhang H: MiR-99a suppressed cell proliferation and invasion by directly targeting HOXA1 through regulation of the AKT/mTOR signaling pathway and EMT in ovarian cancer. Eur Rev Med Pharmacol Sci 2019, 23(11):4663-4672. 
11. Garrido-Cano I, Pla L, Santiago-Felipe S, Simón S, Ortega B, Bermejo B, Lluch A, Cejalvo JM, Eroles P, Martínez-Máñez R: Nanoporous Anodic Alumina-Based Sensor for miR-99a-5p Detection as an Effective Early Breast Cancer Diagnostic Tool. ACS Sens 2021, 6(3):1022-1029.

12. Pradhan V, Hire H, Ghosh K, Singh R, Tiwari IM, Jagadeesh H, Kansal R, Gupta R, Koundal K, Saini $\mathrm{R}$ : MicroRNA profile in understanding pathogenesis of systemic lupus erythematosus NISCAIR-CSIR, India 2012, 21:129-133.

13. Fengzhi J: Identification of microRNA associated with systemic lupus erythematosus in Korean. Ajou University; 2014.

14. Frangou EA, Bertsias GK, Boumpas DT: Gene expression and regulation in systemic lupus erythematosus. European Journal of Clinical Investigation 2013, 10(43):1084-1096.

15. Ofek $\mathrm{P}$, Tiram $\mathrm{G}$, Satchi-Fainaro R: Angiogenesis regulation by nanocarriers bearing RNA interference. Adv Drug Deliv Rev 2017, 119.

16. Wu X-n, Ye Y-x, Niu J-w, Li Y, Li X, You X, Chen H, Zhao L-d, Zeng X-f, Zhang F-c et al: Defective PTEN regulation contributes to $B$ cell hyperresponsiveness in systemic lupus erythematosus. Sci Trans/ Med 2014, 6(246):99.

17. Zhang J, Liu Y, Shi G: The circRNA-miRNA-mRNA regulatory network in systemic lupus erythematosus. Clin Rheumatol 2021, 40(1):331-339.

18. Latini A, Ciccacci C, Benedittis GD, Novelli L, Ceccarelli F, Conti F, Novelli G, Perricone C, Borgiani P: Altered expression of miR-142, miR-155, miR-499a and of their putative common target in systemic lupus erythematosus. Epigenomics 2021, 13(1):5-13.

19. Tao B, Xiang W, Li X, He C, Chen L, Xia X, Peng T, Peng L, Yang X, Zhong C: Regulation of Toll-like receptor-mediated inflammatory response by microRNA-152-3p-mediated demethylation of MyD88 in systemic lupus erythematosus. Inflammation Research 2021, 70(3):285-296.

20. Wang T, Mei Y, Li Z: Research Progress on Regulatory B Cells in Systemic Lupus Erythematosus. Biomed Res Int 2019, 2019:7948687.

21. Lin TA, Kong X, Saltiel AR, Blackshear PJ, Lawrence JC: Control of PHAS-I by insulin in 3T3-L1 adipocytes. Synthesis, degradation, and phosphorylation by a rapamycin-sensitive and mitogen-activated protein kinase-independent pathway. The Journal of biological chemistry 1995, 270(31):18531-18538.

22. Long J, He Q, Yin Y, Lei X, Li Z, Zhu W: The effect of miRNA and autophagy on colorectal cancer. Cell Prolif 2020, 53(10):1-10.

23. Sharma P, Dando I, Strippoli R, Kumar S, Somoza A, Cordani M, Tafani M: Nanomaterials for Autophagy-Related miRNA-34a Delivery in Cancer Treatment. Front Pharmaco/ 2020, 11:1141. 
24. Barroso-Chinea P, Luis-Ravelo D, Fumagallo-Reading F, Castro-Hernandez J, Salas-Hernandez J, Rodriguez-Nuñez J, Febles-Casquero A, Cruz-Muros I, Afonso-Oramas D, Abreu-Gonzalez P et al: DRD3 (dopamine receptor D3) but not DRD2 activates autophagy through MTORC1 inhibition preserving protein synthesis. Autophagy 2020, 16(7):1279-1295.

25. Brier LW, Ge L, Stjepanovic G, Thelen AM, Hurley JH, Schekman R: Regulation of LC3 lipidation by the autophagy-specific class III phosphatidylinositol-3 kinase complex. Mol Biol Cel/ 2019, 30(9):10981107.

26. Peng J-Q, Han S-M, Chen Z-H, Yang J, Pei Y-Q, Bao C, Qiao L, Chen W-Q, Liu B: Chaperonemediated autophagy regulates apoptosis and the proliferation of colon carcinoma cells. Biochem Biophys Res Commun 2020, 522(2):348-354.

27. Arkatkar T, Jacobs HM, Du SW, Li Q-Z, Hudkins KL, Alpers CE, Rawlings DJ, Jackson SW: TACI deletion protects against progressive murine lupus nephritis induced by BAFF overexpression. Kidney Int 2018, 94(4):728-740.

28. Tanaka Y: State-of-the-art treatment of systemic lupus erythematosus. Int J Rheum Dis 2020, 23(4):465-471.

29. Kim H, Kim T, Kim M, Lee HY, Kim Y, Kang MS, Kim J: Activation of the alternative complement pathway predicts renal outcome in patients with lupus nephritis. Lupus 2020, 29(8):862-871.

30. López P, Rodríguez-Carrio J, Martínez-Zapico A, Pérez-Álvarez Ál, Benavente L, Caminal-Montero L, Suárez A: IgM anti-phosphorylcholine antibodies associate with senescent and IL-17+ T cells in SLE patients with a pro-inflammatory lipid profile. Rheumatology (Oxford) 2020, 59(2):407-417.

\section{Figures}




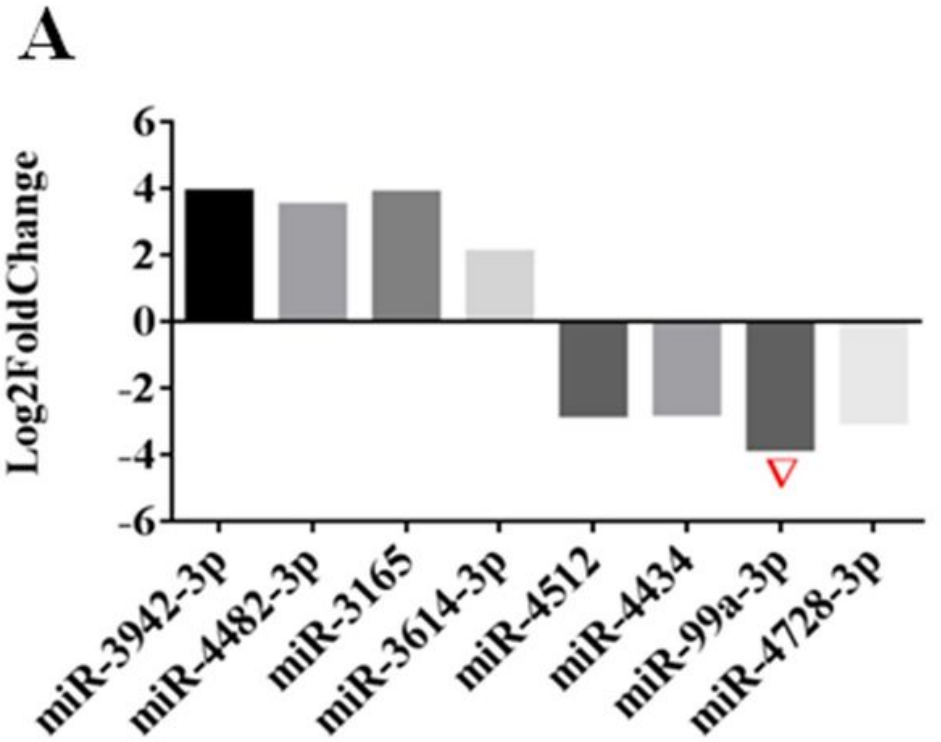

Gene

C

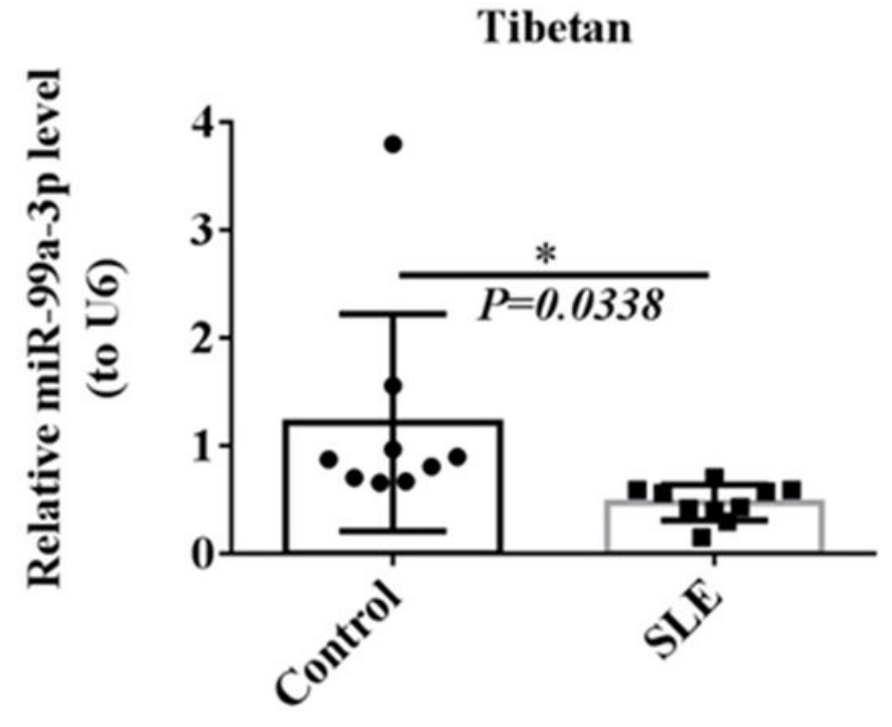

\section{B}

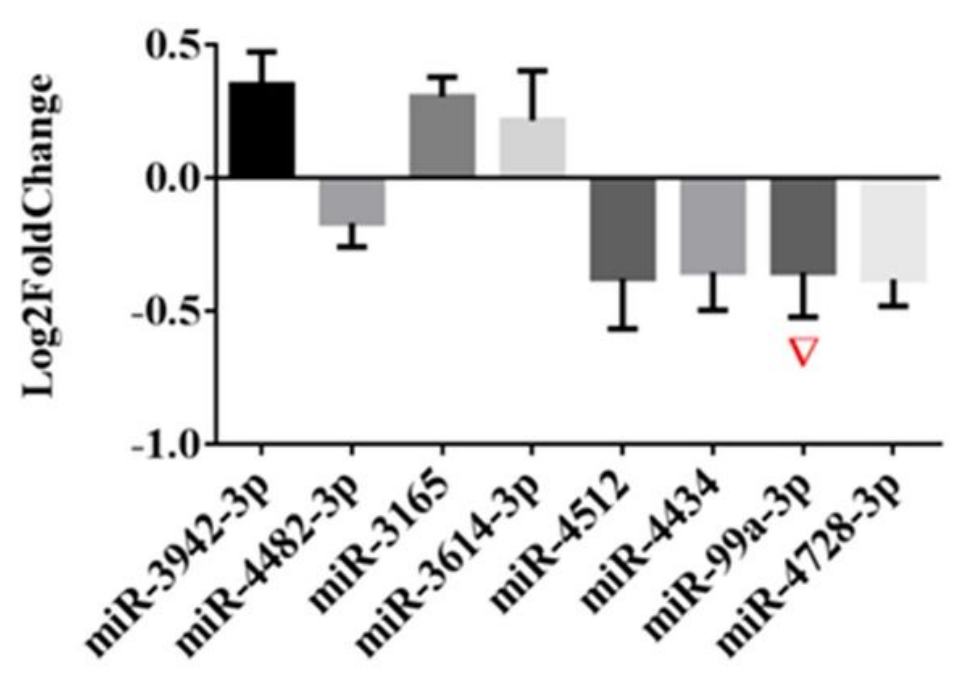

Gene

D

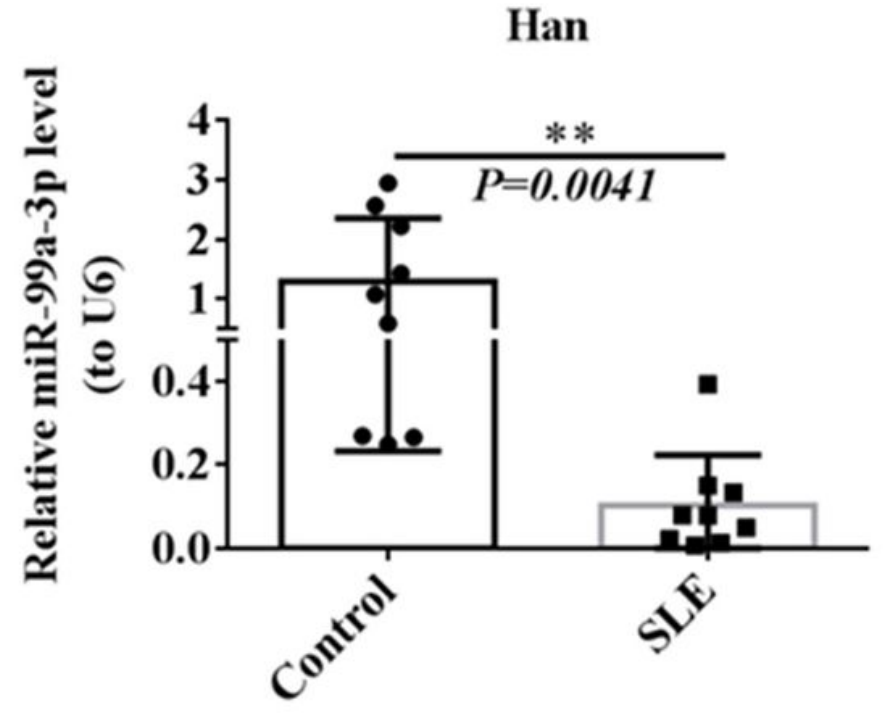

Figure 1

Differential verification of miR-99a-3p in SLE. (A) Screening of significantly differentially expressed miRNAs by second-generation high-throughput sequencing (B) RT-qPCR verification of significantly differentially expressed miRNAs (C, D) RT-qPCR detected miR-99a-3p in PBMCs of Tibetan\&Han SLE patients and Tibetan\&Han healthy individulas. 
A

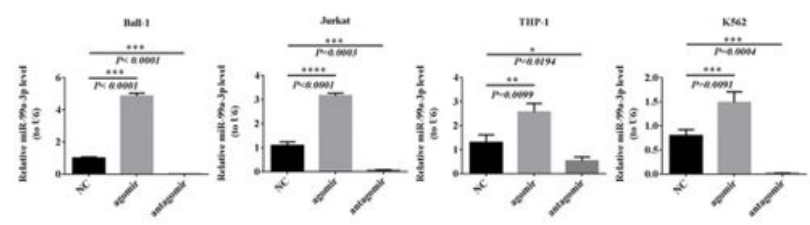

C

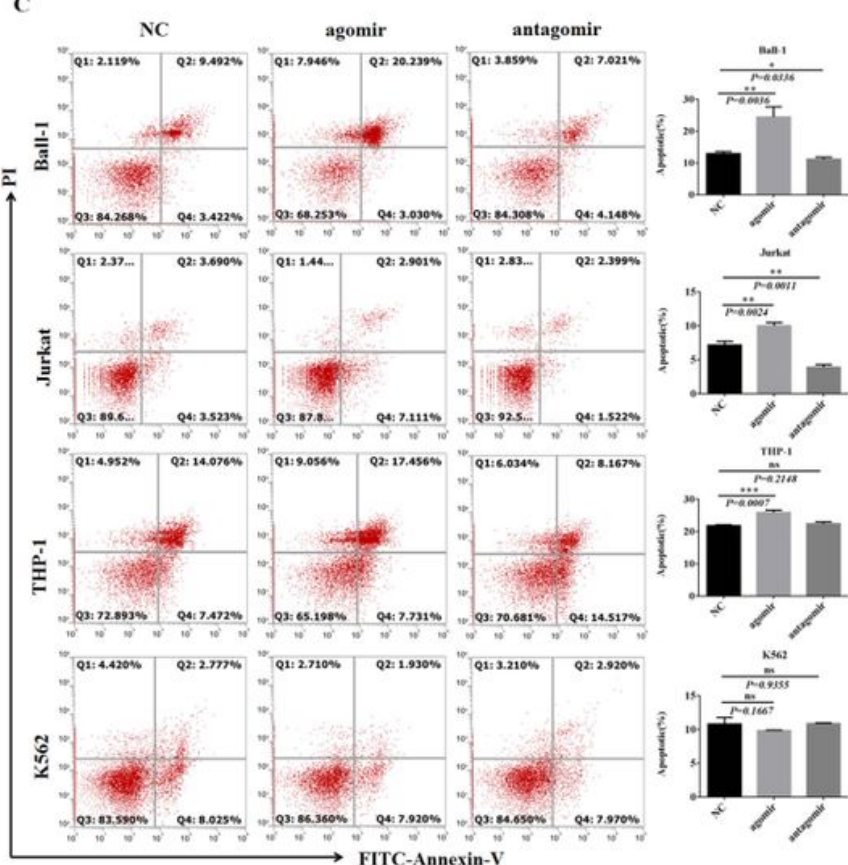

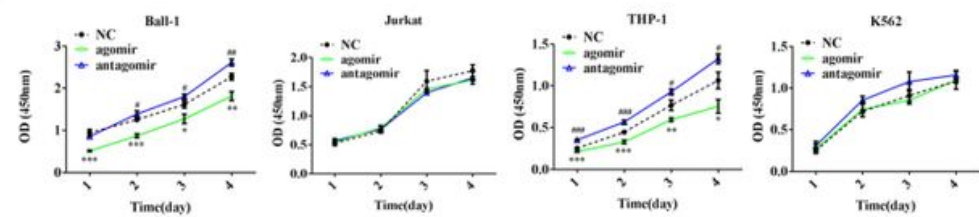

D
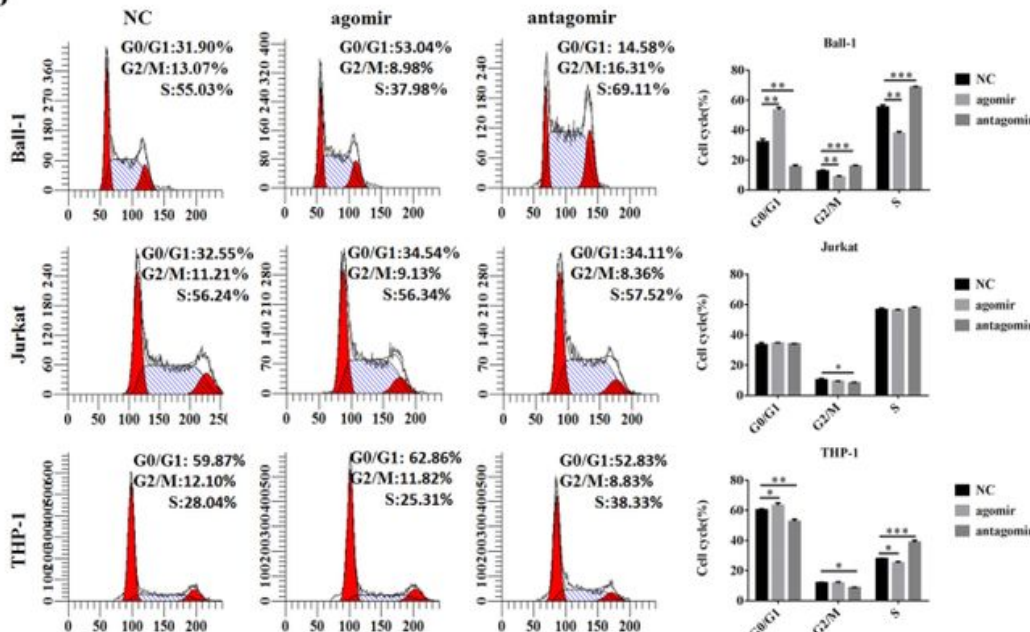

${ }_{\theta}^{200}$
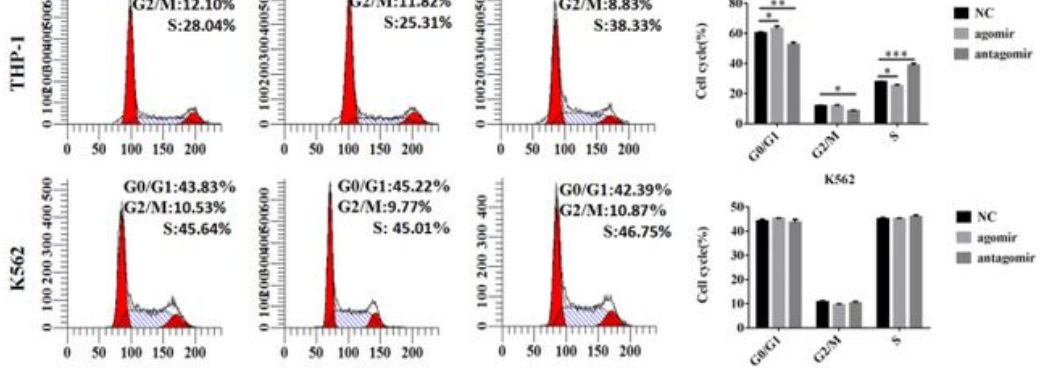

Figure 2

The functional difference of miR-99a-3p on Ball-1, Jurkat, THP-1 and K562 cells. (A) miR-99a-3p expression after miR-99a-3p agomir, antagomir, NC transfected in different cells (B) CCK-8 method to detect the differences in cell proliferation changes on 1,2,3, and 4 days after miR-99a-3p agomir, antagomir, and NC transfected with different cells(C) After miR-99a-3p agomir, antagomir, and NC transfected different cells, flow cytometry was used to analyze the changes in apoptosis of each cell and the histogram to analyze the differences in the proportion of apoptosis in each group (Q1 necrosis, Q2 late apoptosis, Q3 normal, Q4 early apoptosis) (D) miR-99a-3p agomir, antagomir, and NC were transfected with different cells to analyze the cycle changes of each cell by flow cytometry and the histogram to compare differences in the ratio of cells (Note in Fig $B:{ }^{*} P<0.05, * * P<0.01,{ }^{* \star *} P<0.001$, comparison between agomir and $\mathrm{NC}$; $\mathrm{P}<0.05, \# \# \mathrm{P}<0.01$,\#\#\#P<0.001, antagomir and NC Comparison; in Fig $\left.D:{ }^{*}<<0.05,{ }^{* \star} P<0.01,{ }^{\star \star *} P<0.001\right)$ 
A

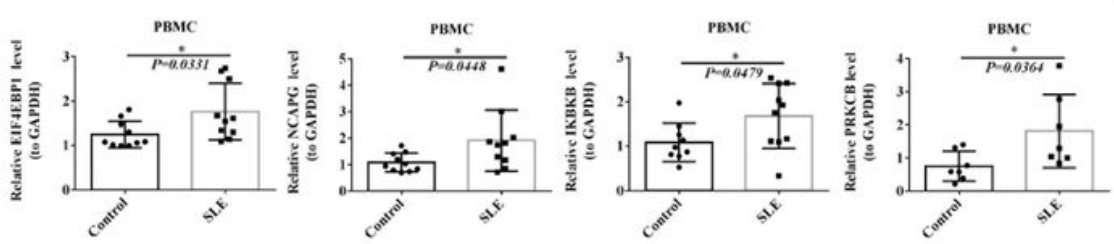

B

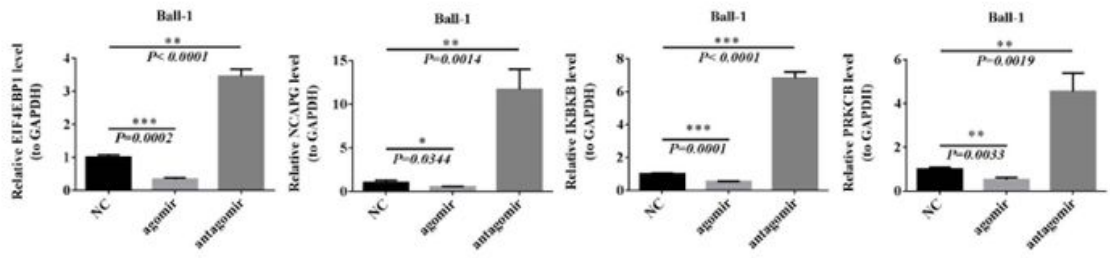

C

EIF4EBP1-wt: 5' UUGUGUAUUCUCAAAGAGCUUAC3' | | | | | hsa-miR-99a-3p: 3' GUCUGGGUAUCUUCGCUCGAAC 5'

EIF4EBP 1-mt: 5' UUGUGUAUUCUCAAACUCGAAAC 3'

NCAPG-wt: 5' AAACUGGCUAGAAAGGAGCUUAU3' $11111+111111$ hsa-miR-99a-3p: 3' GUCUGGGUAUCUUCGCUCGAAC 5'

NCAPG-mt: 5' AAACUGGCAUCUUAGCUCGAAAU 3' IKBKB-wt: 5' UGGGCUAAGGGCUGUGAGCUUAU3' | | | | | |

hsa-miR-99a-3p: 3' GUCUGGGUAUCUUCGCUCGAAC 5'

IKBKB-mt: 5' UGGGCUAAGGGCUGUCUCGAAAU 3' PRKCB-wt: 5' AAAGAGGAGGCAUUCGAGCUUU3' | | | | | | | hsa-miR-99a-3p: 3' GUCUGGGUAUCUUCGCUCGAAC 5'

PRKCB-mt: 5' AAAGAGGAGGCAUUGCUCGAAU 3'

D
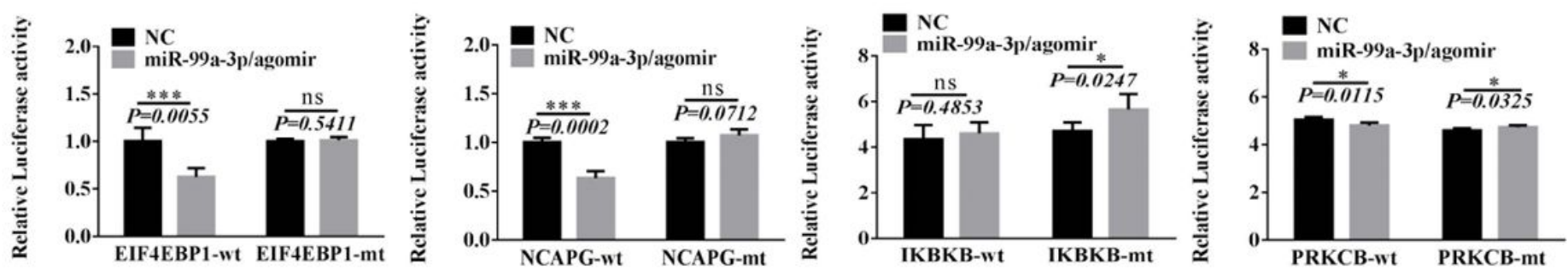

$\mathbf{E}$
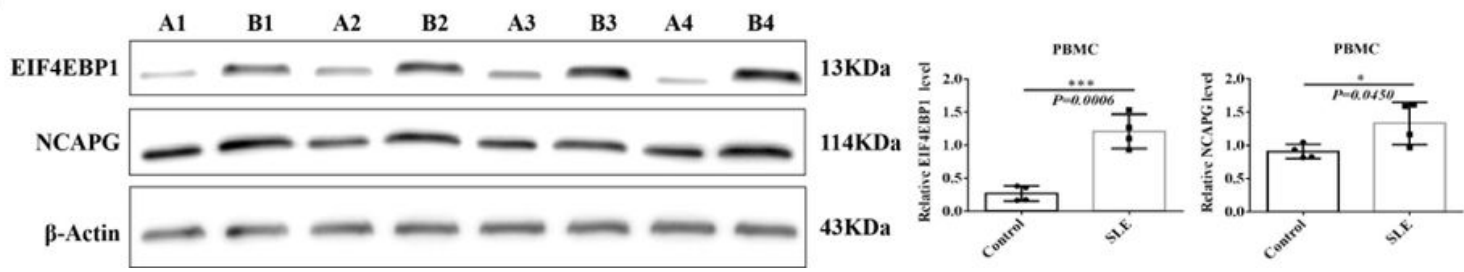

Figure 3

Target gene confirmation. (A)RT-qPCR detect the predicted target gene expression between SLE and healthy individulas(B)Changes in target genes after miR-99a-3p agomir, antagomir, and NC transfected Ball-1 cells(C) Prediction of binding site of miR-99a-3p and target gene and mutation construction(D) Transfect 293T cells with mutant and wild-type fluorescein vectors of binding sites and agomir, read the fluorescein report value through a fluorescence spectrophotometer, and analyze the difference in the influence of agomir between mutant and wild-type $(E)$ Western blotting verified the difference in expression of EIF4EBP1, NCAPG in SLE (B1, B2, B3, B4) and healthy individulas (A1, A2, A3, A4). 
A

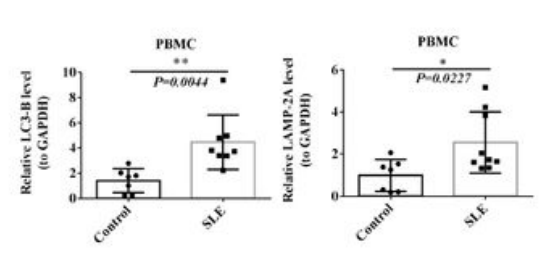

C

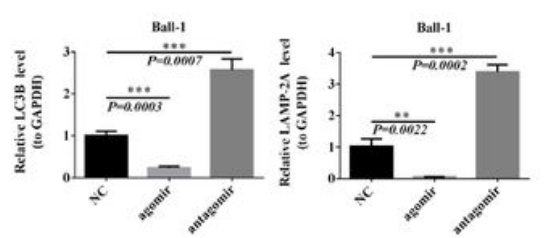

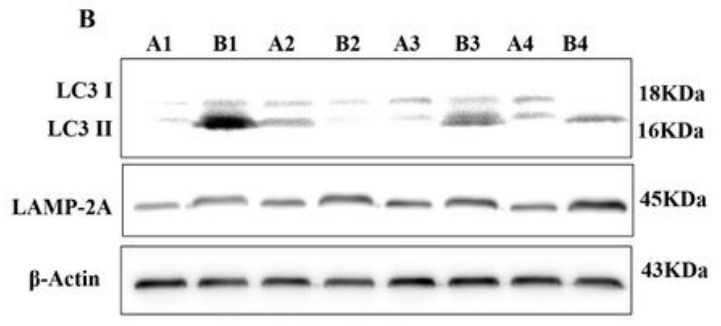

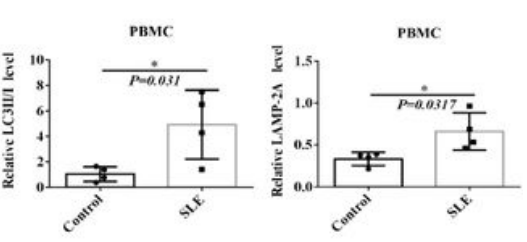

D
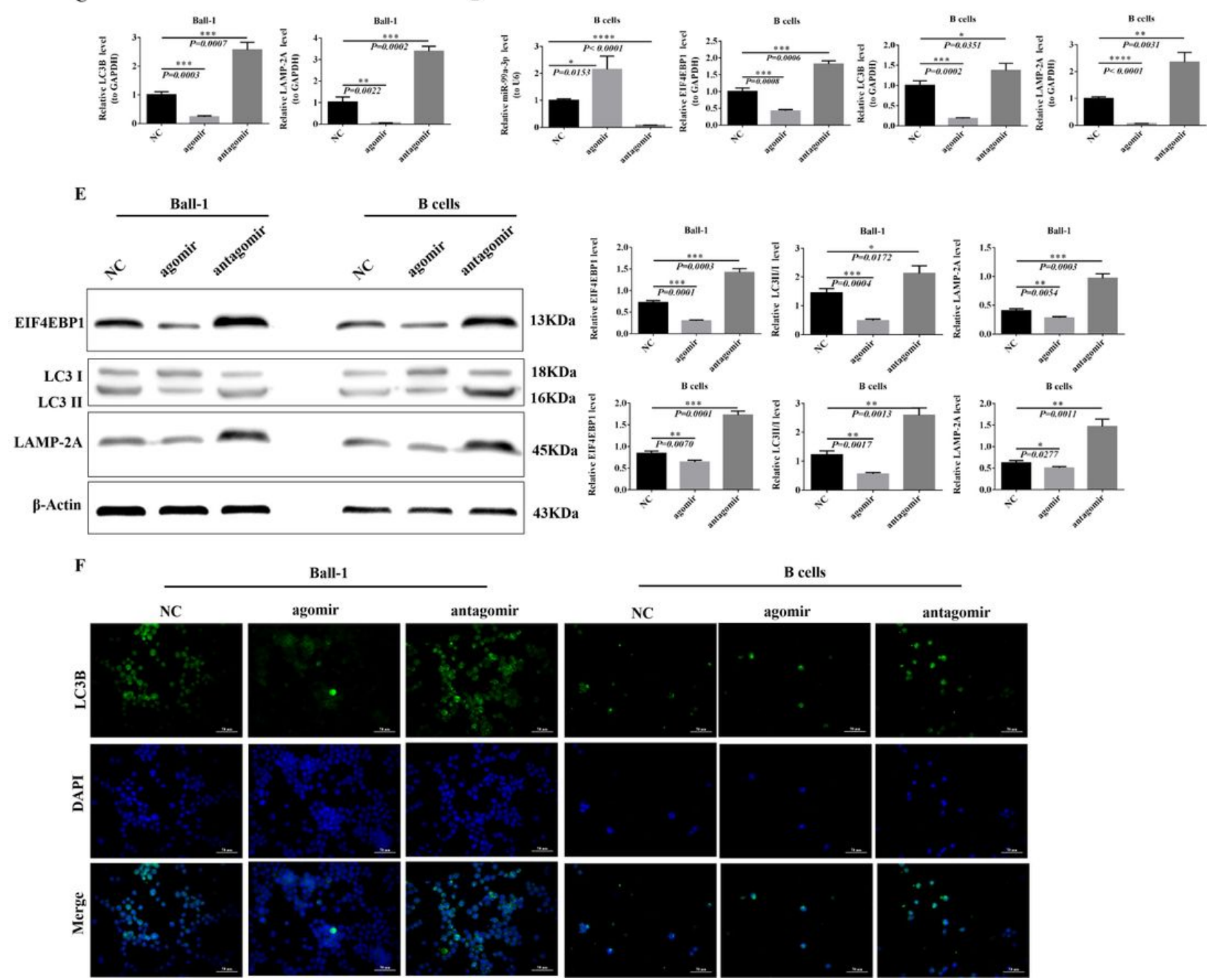

Ball-1
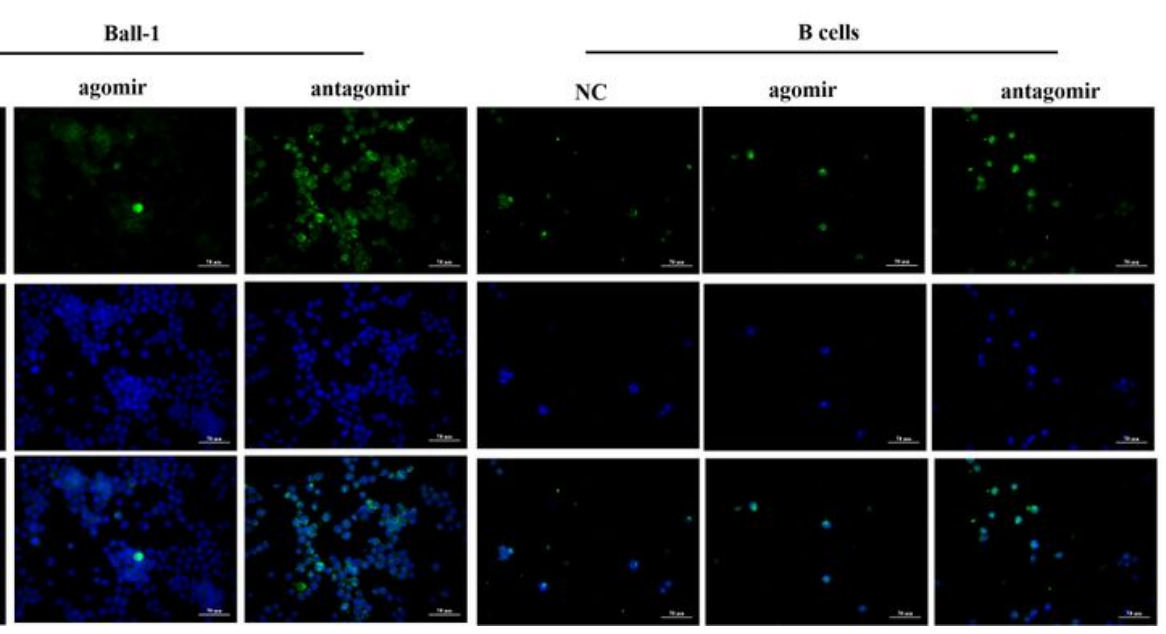

\section{Figure 4}

miR-99a-3p participates in the autophagy signaling pathway through target genes. $\triangle A \triangle R T-q P C R$ detected

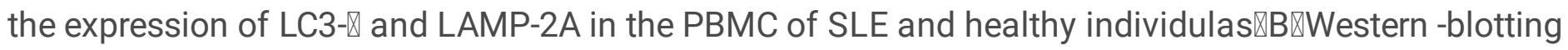
detected the expression of LC3-囚, LAMP-2A in SLE (B1, B2, B3, B4) and healthy individulas (A1, A2, A3, A4) $\triangle C \triangle R T-q P C R$ detected of the expression of LC3- $\triangle$ and LAMP-2A after miR-99a-3p agomir, antagomir and NC transfected Ball-1هD $\triangle R T-q P C R$ detected of the expression of miR-99a-3p, EIF4EBP1, LC3- $\triangle$ and LAMP$2 A$ after miR-99a-3p agomir, antagomir and NC transfected B cells囚E $\triangle$ Western-blotting detected the expression of EIF4EBP1, LC3- $\$ and LAMP-2A protein after miR-99a-3p agomir, antagomir, NC transfected

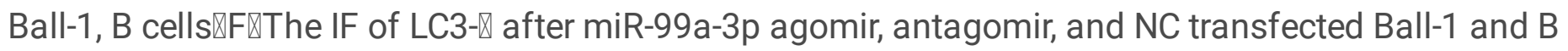




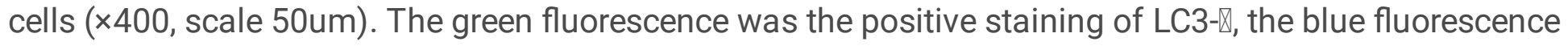
was the nuclear staining of DAPI, and the Merge fluorescence was the image of the fusion of LC3- $\triangle$ and DAPI.

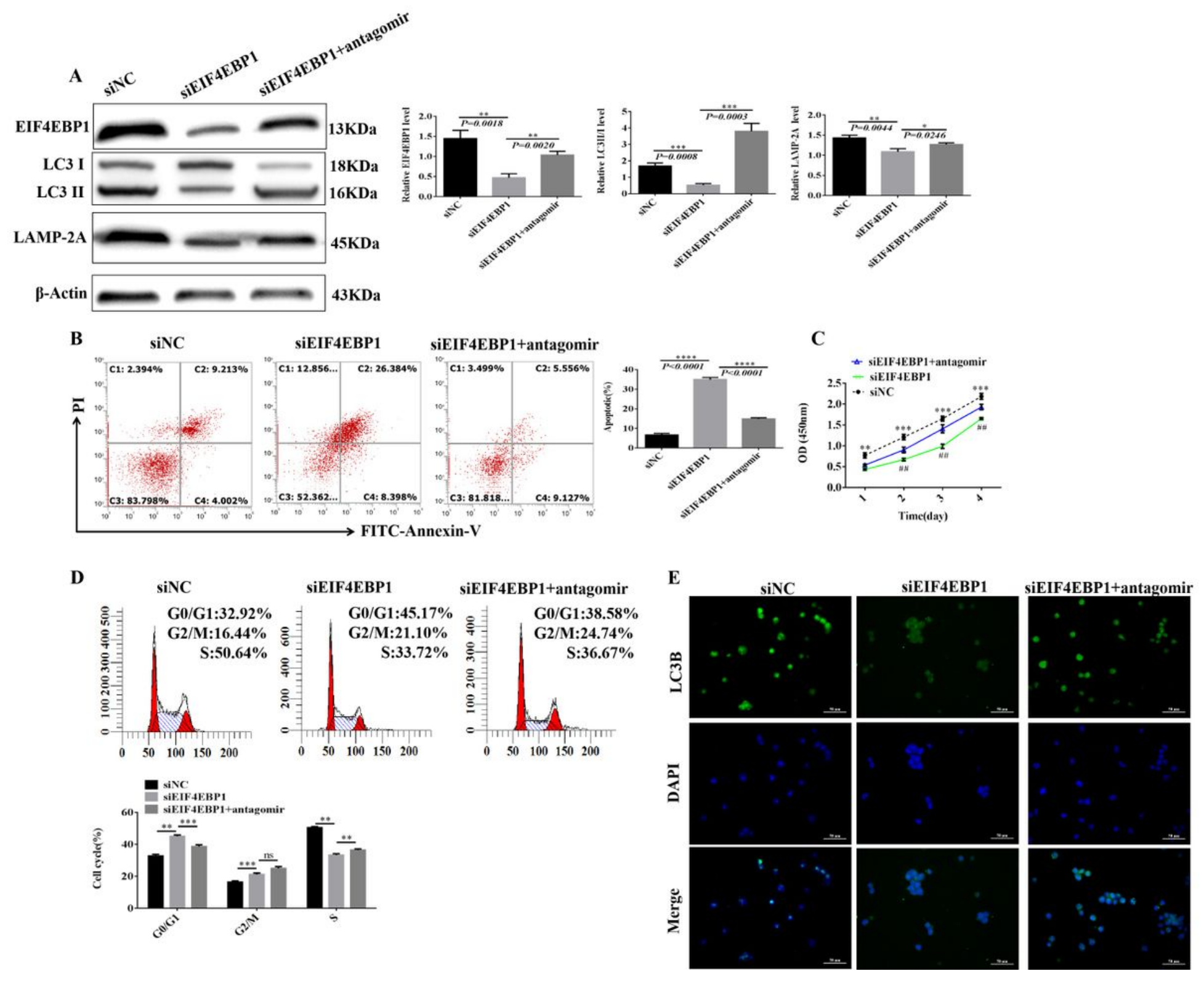

\section{Figure 5}

Ball-1 function changes after Rescue experiment. $\ A \rrbracket W e s t e r n$ blotting was used to detect the expression

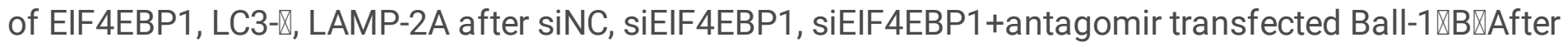
siNC, siEIF4EBP1, siEIF4EBP1+antagomir were transfected with Ball-1, flow cytometry was used to analyze the changes in apoptosis of each cell line and the histogram to analyze the differences in the proportion of apoptosis in each group(C1 necrosis, C2 late apoptosis, C3 normal, C4 early apoptosis) $\backslash \mathrm{C} \bigotimes$ CCK-8 method to detect the difference in cell proliferation of siNC, siEIF4EBP1, siEIF4EBP1+antagomir transfected with Ball-1 at 1, 2, 3, and 4 days $\triangle \mathrm{D} \bigotimes$ After siNC, siEIF4EBP1, siEIF4EBP1+antagomir were transfected into Ball-1, flow cytometry was used to analyze the differences in the cycle changes of each cell and the histogram to compare the differences in the proportion of cells in each group. $\mathrm{EE} \otimes T$ The IF of 
LC3- $\$ after siNC, siEIF4EBP1, siEIF4EBP1+antagomir transfected Ball-1 ( $\times 400$, scale 50um). Green fluorescence was the positive staining of LC3-囚, blue fluorescence was the nuclear staining of DAPI, and

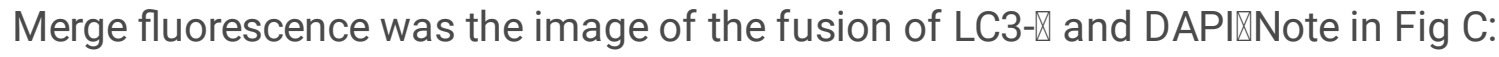
$\star \star P<0.01,{ }^{* \star * P}<<0.001$, comparison between siEIF4EBP1 and siNC; \#\#P<0.01,comparison between

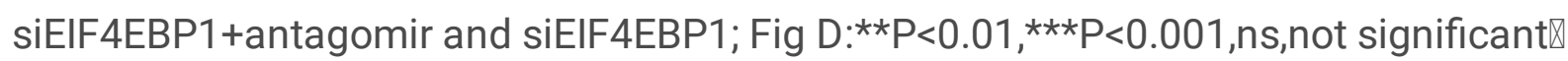

A

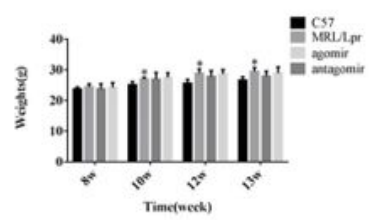

B

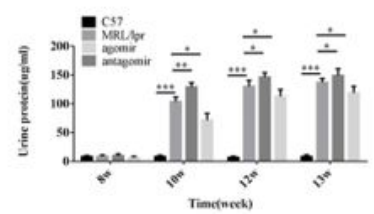

C

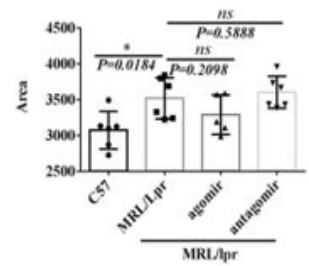

D

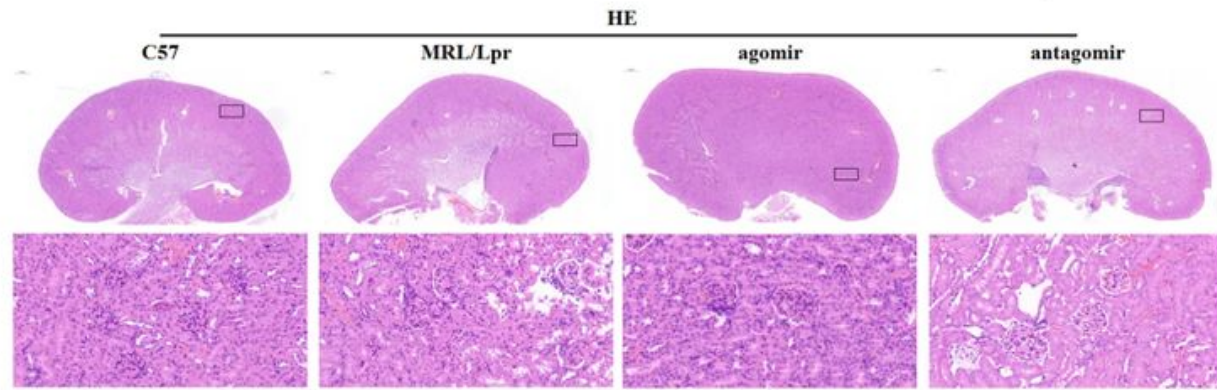

E

C57
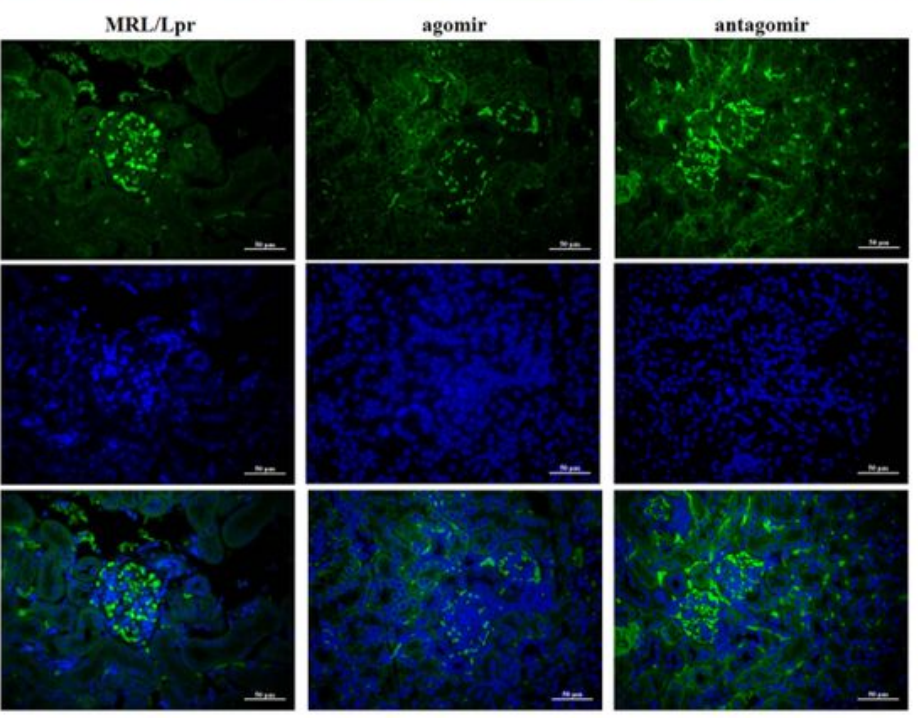

F

G
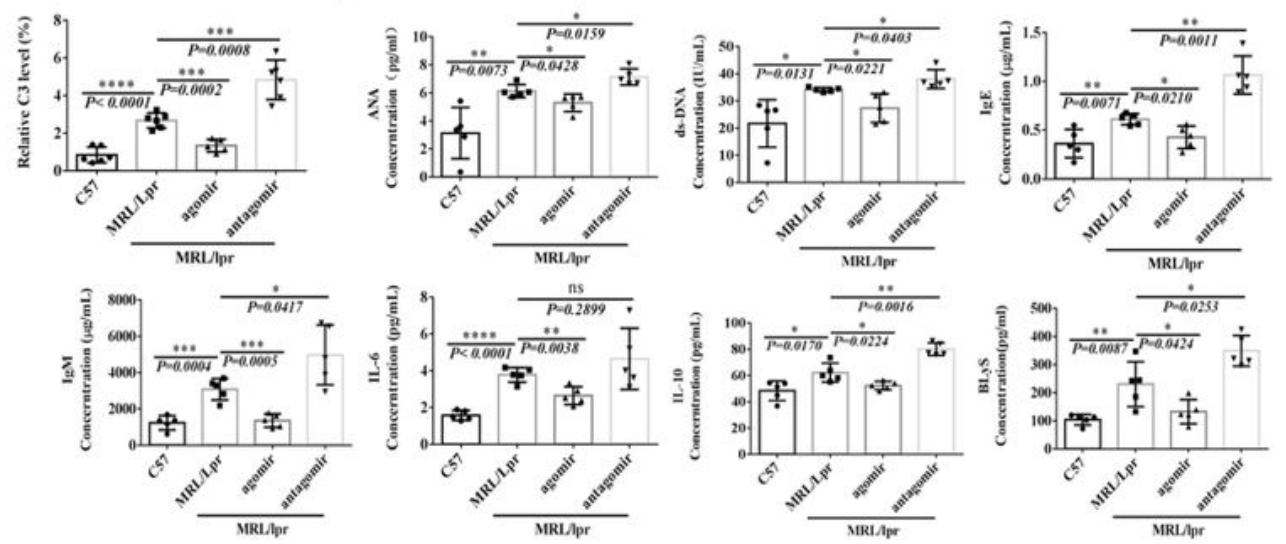

Figure 6 
Disease progression of MRL/Ipr lupus mice after experimental intervention of miR-99a-3p in vivo. $₫ A \rrbracket 8 w$, $10 \mathrm{w}, 12 \mathrm{w}, 13 \mathrm{w}$ body weight changes after intervention of MRL/Ipr and C57 mice $\mathbb{B} \llbracket C o o m a s s i e$ brilliant blue method to detect the changes of $8 \mathrm{w}, 10 \mathrm{w}, 12 \mathrm{w}, 13 \mathrm{w}$ urine protein after intervention of MRL/Ipr and C57 mice $\mathbb{C} \varangle \mathrm{HE}$ staining of glomerular area after intervention of MRL/Ipr and C57 mice $\mathbb{D} \otimes H E$ staining of $\mathrm{MRL} / \mathrm{Ipr}$ and C57 mice after intervention (×2.5,×400)区E $\mathrm{TThe}$ C3 IF of MRL/Ipr and C57 mice after intervention ( $\times 400$, scale 50um). Green fluorescence was C3 positive staining, blue fluorescence was DAPI nuclear staining, and Merge fluorescence was the image after fusion of C3 and DAPI\&F『C3 IF after intervention of MRL/Ipr and C57 mice区G区ELISA detected ANA, dsDNA, IgE, IgM, IL-6, IL-10, BLyS

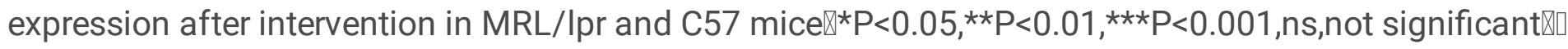

A
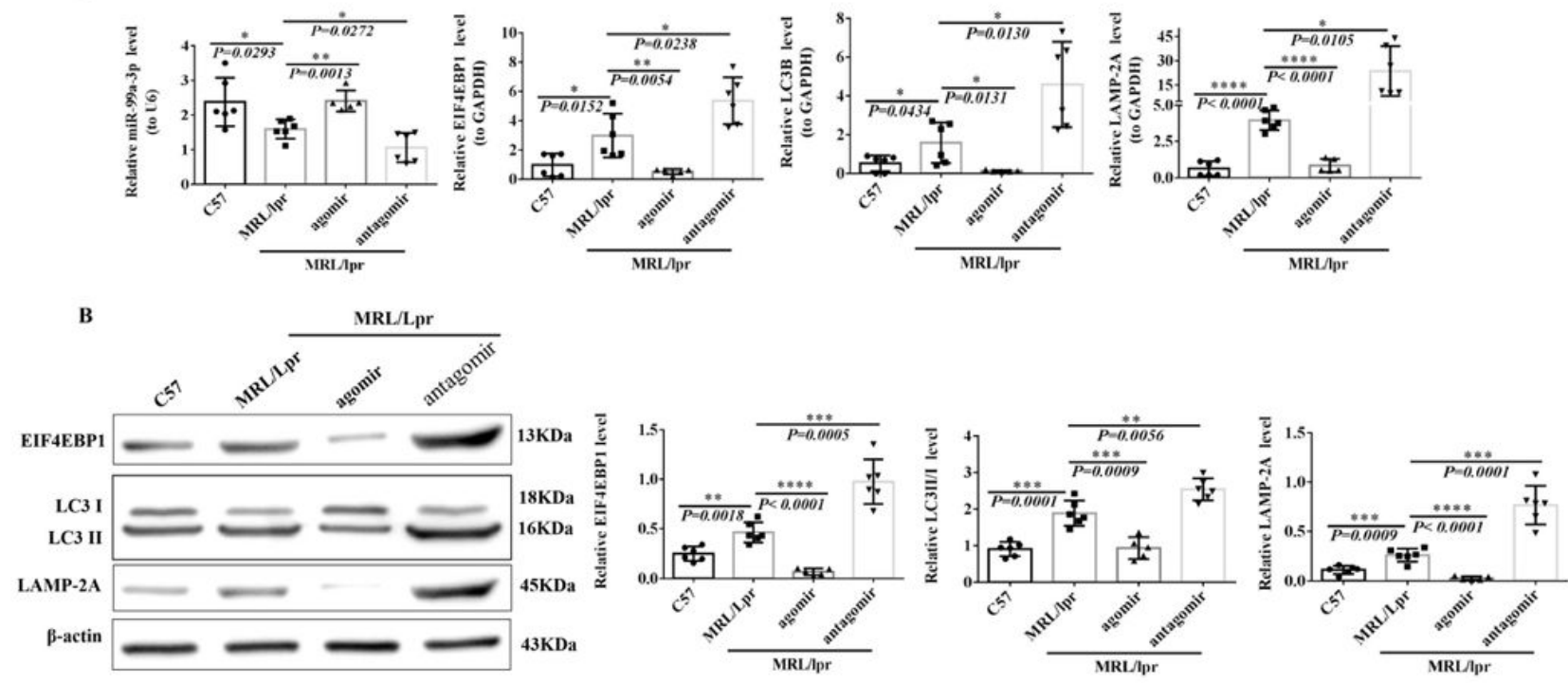

C
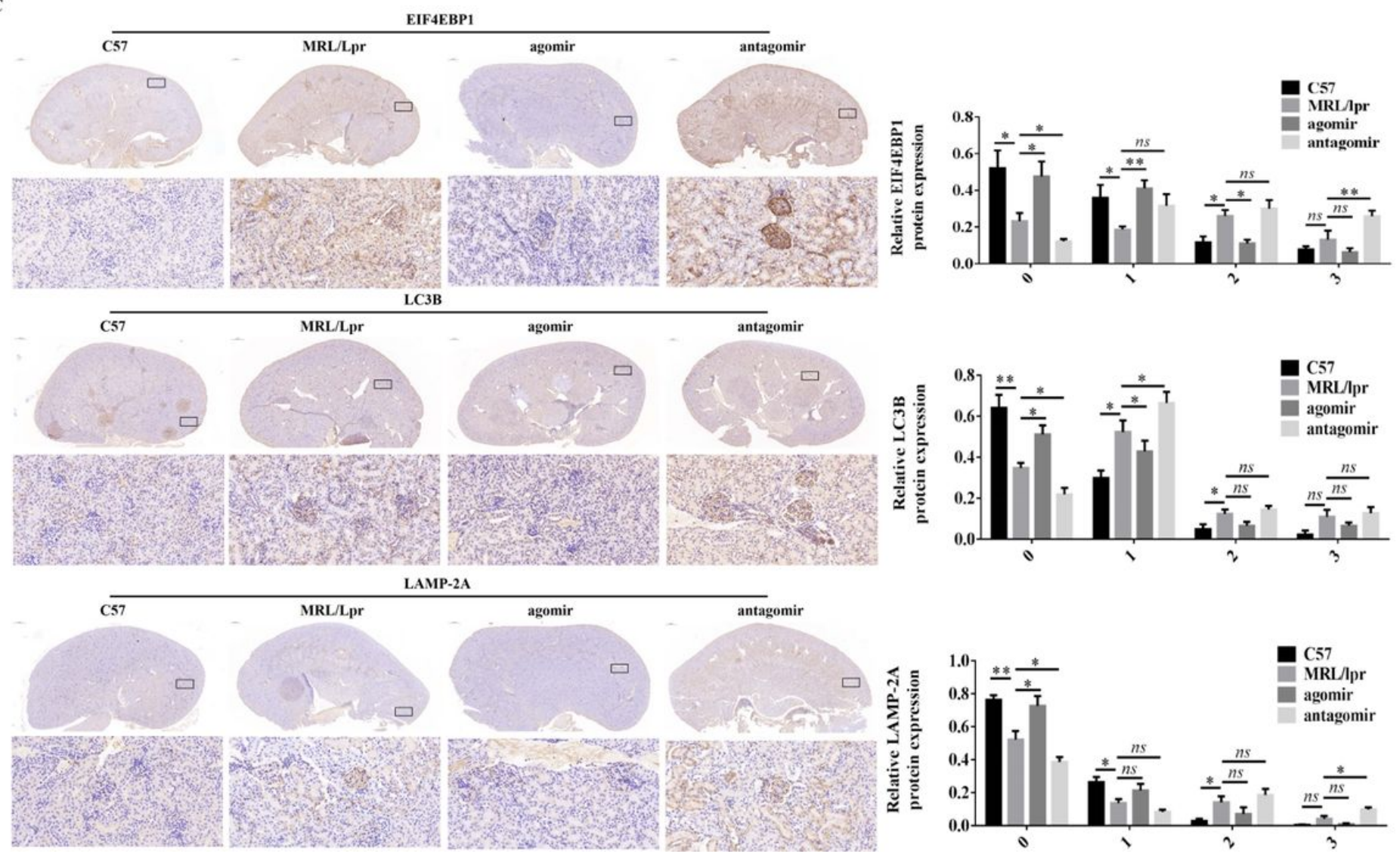


\section{Figure 7}

Changes of target genes and pathway proteins in MRL/Ipr lupus mice after experimental intervention of miR-99a-3p in vivo $\triangle A \triangle R T-q P C R$ detection of miR-99a-3p, EIF4EBP1, LC3-囚, LAMP-2A changes after intervention $₫ \mathrm{~B} \otimes$ Western-blotting detection of EIF4EBP1, LC3-邓, LAMP-2A protein expression after

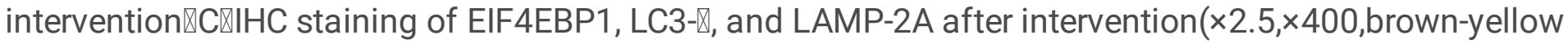
was positive staining, ${ }^{*} P<0.05,{ }^{*} P<0.01, n s$, not significant $\triangle$

\section{Supplementary Files}

This is a list of supplementary files associated with this preprint. Click to download.

- Supplementarymaterials.docx 